\title{
Heat Transfer in a Fractional Nanofluid Flow through a Permeable Medium
}

\author{
Muhammad Shoaib Anwar (D), ${ }^{1}$ Muhammad Irfan, ${ }^{2}$ Majid Hussain, ${ }^{3}$ Taseer Muhammad, ${ }^{4}$ \\ and Zakir Hussain ${ }^{5}$ \\ ${ }^{1}$ Department of Mathematics, University of Jhang, Gojra Road, Jhang 35200, Pakistan \\ ${ }^{2}$ Department of Mathematics, University of WAH, Quaid Avenue, Wah Rawalpindi, Punjab 47040, Pakistan \\ ${ }^{3}$ Department of Natural Sciences and Humanities, University of Engineering and Technology, Lahore 54890, Pakistan \\ ${ }^{4}$ Department of Mathematics, College of Sciences, King Khalid University, Abha 61413, Saudi Arabia \\ ${ }^{5}$ Department of Mathematics, University of Baltistan, Skardu, Pakistan
}

Correspondence should be addressed to Muhammad Shoaib Anwar; shoaib_tts@yahoo.com

Received 19 October 2021; Revised 6 December 2021; Accepted 28 January 2022; Published 2 March 2022

Academic Editor: Ali Ahmadian

Copyright (c) 2022 Muhammad Shoaib Anwar et al. This is an open access article distributed under the Creative Commons Attribution License, which permits unrestricted use, distribution, and reproduction in any medium, provided the original work is properly cited.

\begin{abstract}
This study examines viscoelastic fractional nanofluid flow through Darcy medium. Memory characteristics due to elasticity are explored with noninteger time derivatives. The unsteady motion of MHD flow is modeled by nonlinear differential equations. Buoyancy forces are incorporated via convection parameters in the flow domain. Fractional relaxation time is considered to control the propagation speed of temperature. A finite difference, along with finite element, a numerical algorithm has been developed for the computation of governing flow equations. Friction coefficient, Sherwood numbers, and Nusselt numbers are computed for the noninteger derivative model. Simulations revealed that noninteger numbers have congruous behavior for concentration, temperature, and velocity fields. It is also noted that heat flux, $\delta_{1}$, and mass flux, $\delta_{2}$, numbers have contradictory effects on the friction coefficient. Various flows, particularly in polymer industries and electrospinning for the production of nanofibers, can be tackled in a comparable pattern.
\end{abstract}

\section{Introduction}

In this communication, we have described the transport of momentum, heat, and concentration with the help of mathematical relations. Mathematical relations are formulated with constitutive expressions that handle fluxes of the above-prescribed quantities [1]. Formulated equations, remained helpful to analyze the transport of heat, diffusion of chemical species, movement of geological flows, engineering applications, meteorology, material science, and medicines [2, 3]. Molecular contact and Brownian motion [1] characterized the conductivity, diffusivity, and viscosity for the nanofluid in the flow domain. Particularly, transfer of heat is an analog of mass transfer in the constitutive expressions for fluxes. Fick's law governs the diffusion in mass transport, and Fourier's law handles the conduction mechanism in heat transfer. Here we have considered the generalized Fourier and Fick laws to incorporate relaxation times. Transport phenomena in Newtonian and nonNewtonian flows can be seen in heat exchangers, thermal devices, granular insulation, fiber technology, nuclear repositories, fermentation processes, geothermal extractions, and the production of crude oil [4-11]. In the literature, the transfer of heat and mass is widely reported by distinguished researchers. For example, Salama et al. [12] discussed the solution of a flow problem through porous media with flux approximations. In this communication, the viscoelastic fluid model of second grade is analyzed for the transport mechanism with suspended nanoparticles. Viscoelastic second-grade fluid model exhibits differential type nature. For differential fluids, stress is expressed by an explicit velocity gradient. Differential models describe the fluids in 
terms of their shear thickening and thinning effects, normal stress effects, thixotropy exhibition, and nonlinear behavior of creeping and yielding. Flow problems, which include polymer suspensions and slurries, in fiber handling can be discussed with a given mathematical model [13].

Governing flow equations with noninteger time derivatives have been proven for useful analysis of viscoelastic trends [14]. Nowadays, mathematical modeling with noninteger derivatives have gained importance in various branches of science. For example, valuable impact can be seen in fluid mechanics. Moreover, the influence of noninteger modeling is seen in viscoelasticity, control theory, and electrochemistry [15-17]. Advancements in fractional modeling are carried out with the passage of time and the inclusion of new fractional derivatives in the literature, for example, Yang's fractional derivative, Atangana-Baleanu derivatives, and Caputo derivatives $[18,19]$. Both natural and artificial systems can be analyzed in a better way via noninteger time derivatives as they describe hereditary aspects of materials [20-24]. Simulations of anomalous nonlinear models can be used to approximate experimental data in a better way when compared to usual rheological models [25-30].

Porous medium flows occur in soil mechanics, ceramics, and industrial and mineral processing. The complexity of the flow problem is increased with the presence of a porous medium. This is mainly due to the interface between packing particles and fluid molecules. Darcy flow is analyzed by various researchers. Nonlinear Darcy flow is examined by Ervin et al. [31]. The Lorentz force shows a significant role in viscoelastic problems such as in MHD generators and control theory. Magnetic field impacts can be seen in porous medium flows that are encountered in metallurgical systems, extrusion and penetration practices, etc. The flow domain is greatly influenced by convection as a transport mechanism, is greatly changed by both buoyancy and gravitational forces. Convection's importance can be seen in boilers, nuclear reactors, and heat exchangers. Zhou and Liang [32] discussed convection in unsteady flow problems.

The industrial importance of nanofluids is significant in lightweight materials production, the breakdown of organic pollutants, and in the production of nanofibers $[33,34]$. Here, under consideration, flow is unidirectional through a channel of infinite extent. By this configuration, fluid velocity and its gradient are orthogonal to each other, which may lead to the addition of a nonlinear convection term in the governing equations. These flows are mostly observed in the long narrow channels. Many microchannel flows are described by these solutions, their superposition, and by the small perturbation of these flows $[35,36]$. Viscoelastic flows can also be used in electrospinning for nanofibers production. Nanofibers are formulated by an electric charged jet of polymer solution that is of viscoelastic nature. An increase of yield stress along with a viscoelastic jet causes a decrease in fluid elongation $[37,38]$. The structure of nanoparticles is between atomic and bulk. The possibility of nano particles' inclusion is due to their strong interaction with base fluid. Inclusion of these particles effectively changes the characteristics of the base fluid. For instance, nanoparticle suspension increases the thermal conductivity of the base fluid. Wang and Zhang [39] analyzed heat transfer in nanofluid flow. With the passage of time, fractional nanofluid flows require more attention in literature. Solutions to fractional flow problems are usually discussed with integral transforms. But integral transforms are not helpful for solving nonlinear coupled fractional equations. Due to this, numerical solutions are proposed for flow problems(see [40-43]). In this article, we have tackled the problem with finite element and finite difference techniques to solve the described fractional flow configuration.

Particularly in this communication, we have considered modeling with noninteger derivatives of nanofluid flow. Due to this, we have achieved more control over the flow simulations with the help of fractional derivatives when compared with the classical mathematical flow models. Flux conditions are imposed at $y=0$, while quadratic variations are observed at the fixed boundary. A Darcy flow medium is observed with an applied magnetic field to the flow domain. Moreover, here we have proposed a scheme with numerical discretization to obtain the stable results of the coupled nonlinear fractional equations. Space variable is discretized by finite element while time variable is discretized by finite difference scheme. The influence of the involved physical parameters is discussed appropriately. In Section 2, modeling with noninteger time derivatives is discussed. The flow field is approximated by the proposed scheme in Section 3. A numerical approximation of the flow problem is given in Section 4. Finally, key results are given in Section 5 .

\section{Mathematical Description}

In this study, we considered heat transfer in the MHD flow of the nanoliquid with noninteger derivatives of time. Modeling with noninteger derivatives helps to achieve more control over the flow simulations when compared with the classical models that contain ordinary derivatives. A nanofluid is formed with nanoparticles and base liquid. The medium of fluid flowing is considered to be a Darcy porous medium. Moreover, Neumann boundary conditions are supposed to be in the heating domain. At the start, there is no movement in the medium. At that time, the whole configuration is at constant temperature $\theta_{0}$ and concentration $\phi_{0}$. With the passage of time, disturbance in the liquid is observed due to the lower domain. We consider the velocity field to be a function of $y$ and $t$ only. Then we consider

$$
\mathbf{U}=u(y, t) \mathbf{e}_{\mathbf{x}}
$$

For the Cauchy stress tensor, Rivlin-Ericksen tensors, Darcy law and thermodynamic stability conditions of differential type second grade fluid (see [21] and references therein). The fractional formalism of thermal and concentration gradients can be seen in $[45,46]$.

Here is a brief description of the mathematical modeling. In order to formulate an energy equation with Caputo fractional derivative $\alpha$ including nanoparticles, consider the classical energy equation for incompressible fluid of the Buongiorno article (see [46]). 


$$
\rho_{f} \mathcal{c}_{f} \frac{\partial \theta}{\partial t}=-\nabla \cdot \mathbf{q}+h_{p} \nabla \cdot \mathbf{J}_{p},
$$

where $\mathbf{q}, \mathbf{J}_{p}$ denote fluxes of energy and diffusion and for definition (see [46]). Temperature and concentration propagation of infinite order can be seen with $\mathbf{q}, \mathbf{J}_{p}$ in Buongiorno article (see [46]). We can overcome this situation with the introduction of fractional relaxation times using reference [45], so we define $\mathbf{q}, \mathbf{J}_{p}$ as

$$
\begin{gathered}
\left(1+\frac{\tau_{1}^{\alpha}}{\Gamma(1+\alpha)} \frac{\partial^{\alpha}}{\partial t^{\alpha}}\right) \mathbf{q}=-k \nabla \theta+h_{p}\left(1+\frac{\tau_{1}^{\alpha}}{\Gamma(1+\alpha)} \frac{\partial^{\alpha}}{\partial t^{\alpha}}\right) \mathbf{J}_{p}, \\
\left(1+\frac{\tau_{1}^{\alpha}}{\Gamma(1+\alpha)} \frac{\partial^{\alpha}}{\partial t^{\alpha}}\right) \mathbf{J}_{p}=-\rho_{p} D_{B} \nabla \phi-\rho_{p} D_{\theta} \frac{\nabla \theta}{\theta_{0}} .
\end{gathered}
$$

Now using (2) and (3) and $\nabla h_{p}=c_{p} \nabla \theta[46]$, we obtained

$$
\begin{aligned}
\rho_{f} c_{f}\left(1+\frac{\tau_{1}^{\alpha}}{\Gamma(1+\alpha)} \frac{\partial^{\alpha}}{\partial t^{\alpha}}\right) \frac{\partial \theta}{\partial t}= & k \nabla^{2} \theta+\rho_{p} c_{p}\left(1+\frac{\tau_{1}^{\alpha}}{\Gamma(1+\alpha)} \frac{\partial^{\alpha}}{\partial t^{\alpha}}\right) \\
& \left(D_{B} \nabla \phi \cdot \nabla \theta+\frac{D_{\theta}}{\theta_{0}} \nabla \theta \cdot \nabla \theta\right) .
\end{aligned}
$$

Similarly using the equation of concentration in Buongiorno model [46], we get

$$
\left(\frac{\partial}{\partial t}+\nabla \cdot \mathbf{U}\right) \phi=-\frac{1}{\rho_{p}} \nabla \cdot \mathbf{J}_{p}
$$

reduces to

$$
\left(1+\frac{\tau_{2}^{\beta}}{\Gamma(1+\beta)} \frac{\partial^{\beta}}{\partial t^{\beta}}\right) \frac{\partial \phi}{\partial t}=D_{B} \nabla^{2} \phi+\frac{D_{\theta}}{\theta_{0}} \nabla^{2} \theta .
$$

Finally, governing present flow equations are

$$
\begin{aligned}
& \nabla \cdot \mathbf{U}=0, \\
& \frac{\mathrm{d} \mathbf{U}}{\mathrm{d} t}=\frac{1}{\rho_{f}}(\nabla \cdot \mathbb{S}+\mathbf{r})+\mathbf{b}+g\left[\beta_{1}\left(\theta-\theta_{0}\right)+\beta_{2}\left(\theta-\theta_{0}\right)^{2}\right], \\
& \frac{\mathrm{d}}{\mathrm{d} t}\left(1+\frac{\tau_{1}^{\alpha}}{\Gamma(1+\alpha)} \frac{\partial^{\alpha}}{\partial t^{\alpha}}\right) \theta=\alpha_{3} \frac{\partial^{2} \theta}{\partial y^{2}}+\tau\left(1+\frac{\tau_{1}^{\alpha}}{\Gamma(1+\alpha)} \frac{\partial^{\alpha}}{\partial t^{\alpha}}\right)\left(D_{B} \frac{\partial \theta}{\partial y} \frac{\partial \phi}{\partial y}+\frac{D_{\theta}}{\theta_{0}}\left(\frac{\partial \theta}{\partial y}\right)^{2}\right), \\
& \frac{\mathrm{d}}{\mathrm{d} t}\left(1+\frac{\tau_{2}^{\beta}}{\Gamma(1+\beta)} \frac{\partial^{\beta}}{\partial t^{\beta}}\right) \phi=D_{B} \frac{\partial^{2} \phi}{\partial y^{2}}+\frac{D_{\theta}}{\theta_{0}} \frac{\partial^{2} \theta}{\partial y^{2}},
\end{aligned}
$$

where $\mathbb{S}$ stands for the Cauchy tensor of stress for fluid of second grade (see [21]), $\mathbf{r}$ is the Darcy resistance of porous medium (see [21]), and $\mathbf{b}$ denotes body force.

The pressure gradient is assumed to be negligible.
2.1. Governing Equations. The equations that modeled the flow problem along with their conditions are given here. Continuity (8) will reduce to identity with velocity given in (1). After some mathematical simplifications, modeled equations reduce to

$$
\begin{aligned}
& \frac{\partial u}{\partial t}=\left(v+\frac{\alpha_{1}}{\rho_{f}} \frac{\partial}{\partial t}\right)\left[\frac{\partial^{2} u}{\partial y^{2}}\right]-\frac{\psi}{K}\left(v+\frac{\alpha_{1}}{\rho_{f}} \frac{\partial}{\partial t}\right) u-\frac{\sigma B_{0}^{2}}{\rho_{f}} u+g\left[\beta_{1}\left(\theta-\theta_{0}\right)+\beta_{2}\left(\theta-\theta_{0}\right)^{2}\right], \\
& \frac{\partial}{\partial t}\left(1+\frac{\tau_{1}^{\alpha}}{\Gamma(1+\alpha)} \frac{\partial^{\alpha}}{\partial t^{\alpha}}\right) \theta=\alpha_{3} \frac{\partial^{2} \theta}{\partial y^{2}}+\tau\left(1+\frac{\tau_{1}^{\alpha}}{\Gamma(1+\alpha)} \frac{\partial^{\alpha}}{\partial t^{\alpha}}\right)\left(D_{B} \frac{\partial \theta}{\partial y} \frac{\partial \phi}{\partial y}+\frac{D_{\theta}}{\theta_{0}}\left(\frac{\partial \theta}{\partial y}\right)^{2}\right), \\
& \frac{\partial}{\partial t}\left(1+\frac{\tau_{2}^{\beta}}{\Gamma(1+\beta)} \frac{\partial^{\beta}}{\partial t^{\beta}}\right) \phi=D_{B} \frac{\partial^{2} \phi}{\partial y^{2}}+\frac{D_{\theta}}{\theta_{0}} \frac{\partial^{2} \theta}{\partial y^{2}} .
\end{aligned}
$$


2.1.1. Flow Problem Conditions. Here we are considering the flow in a channel separated by a distance $L$. At the start, the whole flow configuration is still with constant temperature $\theta_{0}$ and constant concentration $\phi_{0}$. Flow is generated by the movement of the domain at $y=0$, while the pressure gradient is assumed to be zero in that case. For temperature and concentration, domain at $y=0$ is connected to a source, and the change in temperature is also changing with time, while in the domain at $y=L$, only temperature changes with time. The case for concentration is similar. Flow conditions are given as

$$
\begin{gathered}
u(0, t)=A \frac{\nu^{2}}{L^{4}} t^{2}, u(L, t)=0, \\
-k\left(\frac{\partial \theta}{\partial y}\right)_{y=0}=\frac{q_{\theta} v^{2}}{L^{4}} t^{2} \text { and } \theta(L, t)=\theta_{0}\left(1+\frac{\nu^{2}}{L^{4}} t^{2}\right), t>0, \\
-D_{B}\left(\frac{\partial \phi}{\partial y}\right)_{y=0}=\frac{q_{\phi} \nu^{2}}{L^{4}} t^{2} \text { and } \phi(L, t)=\phi_{0}\left(1+\frac{v^{2}}{L^{4}} t^{2}\right), t>0, \\
u(y, 0)=0, \theta(y, 0)=\theta_{0}, \phi(y, 0)=\phi_{0} \text { and } \frac{\partial \theta}{\partial t}(y, 0) \\
=0=\frac{\partial \phi}{\partial t}(y, 0),|y| \leq L .
\end{gathered}
$$

A complete description of the flow domain is identified via equations (12)-(18).

2.1.2. Skin Friction Coefficients. Friction among fluid and solid boundaries is of great importance in the analysis of flow domains bounded by solid boundaries. Coefficients of friction are mathematically defined as

$$
\mathscr{C}_{f}:=\frac{2 \tau_{w}}{\rho A^{2}}
$$

where shear stress at the wall is

$$
\tau_{w}=\left[\left(\mu+\alpha_{1} \frac{\partial}{\partial t}\right) \frac{\partial u}{\partial y}\right]_{y=0, L} .
$$

2.1.3. Nusselt Numbers. Change of temperature at $y=0$ and $y=L$ are determined by the dimensionless, Nusselt number. Present flow domain, Nusselt numbers are given as

$$
N u_{1}=\frac{-L(\partial \theta / \partial y)_{y=L}}{\theta_{s_{1}}-\theta_{0}},
$$

where $\theta_{s_{1}}$ is constant temperature, at $y=L$. At $y=0$, the Nusselt number is given by

$$
N u_{2}=\frac{-L(\partial \theta / \partial y)_{y=0}}{\theta_{s_{2}}-\theta_{0}}
$$

here $\theta_{s_{2}}$ is the temperature, at $y=0$.

2.1.4. Mass Transfer Nusselt Numbers. Change of concentration at $y=0$ and $y=L$ is determined by mass transfer Nusselt, Sherwood numbers. The present flow domain and Sherwood numbers are given as

$$
S h_{1}=\frac{-L(\partial \phi / \partial y)_{y=L}}{\phi_{s_{1}}-\phi_{0}}
$$

where $\phi_{s_{1}}$ is the constant concentration at $y=L$. At $y=0$, the Sherwood number is given by

$$
S h_{2}=\frac{-L(\partial \phi / \partial y)_{y=0}}{\phi_{s_{2}}-\phi_{0}}
$$

here $\phi_{s_{2}}$ is the concentration at $y=0$.

2.1.5. Problem in Nondimensionalization. We have defined the following dimensionless quantities to make mathematical problem (12)-(18) as nondimensionalized.

$$
\widehat{y}:=\frac{y}{L}, \widehat{t}:=\frac{v}{L^{2}} t \widehat{u}:=\frac{u}{A}, \widehat{\theta}:=\frac{\theta-\theta_{0}}{\theta_{0}} \widehat{\phi}:=\frac{\phi-\phi_{0}}{\phi_{0}} .
$$

Using, (25), with dimensionless quantities, the IBVP (12)-(18) considered as without hats

$$
\begin{gathered}
(1+\epsilon) \frac{\partial u}{\partial t}=\left(1+\gamma \frac{\partial}{\partial t}\right)\left[\frac{\partial^{2} u}{\partial y^{2}}\right]-(\lambda+H a) u+\left(\lambda_{1}+\lambda_{2} \theta\right) \theta, \\
\operatorname{Pr} \frac{\partial}{\partial t}\left(1+\delta_{3} \frac{\partial^{\alpha}}{\partial t^{\alpha}}\right) \theta=\frac{\partial^{2} \theta}{\partial y^{2}}+\operatorname{Pr}\left(1+\delta_{3} \frac{\partial^{\alpha}}{\partial t^{\alpha}}\right)\left(N b \frac{\partial \theta}{\partial y} \frac{\partial \phi}{\partial y}+N t\left(\frac{\partial \theta}{\partial y}\right)^{2}\right), \\
S c \frac{\partial}{\partial t}\left(1+\delta_{4} \frac{\partial^{\beta}}{\partial t^{\beta}}\right) \phi=\frac{\partial^{2} \phi}{\partial y^{2}}+\left(\frac{N t}{N b}\right) \frac{\partial^{2} \theta}{\partial y^{2}},|y|<1, t>0,
\end{gathered}
$$

and problem conditions are given by 


$$
\left\{\begin{array}{l}
u(0, t)=t^{2}, u(1, t)=0,\left(\frac{\partial \theta}{\partial y}\right)_{y=0}=-\delta_{1} t^{2},\left(\frac{\partial \phi}{\partial y}\right)_{y=0}=-\delta_{2} t^{2}, \theta(1, t)=t^{2}=\phi(1, t), \\
u(y, 0)=0, \theta(y, 0)=0=\frac{\partial \theta}{\partial t}(y, 0) \operatorname{and} \phi(y, 0)=0=\frac{\partial \phi}{\partial t}(y, 0) .
\end{array}\right.
$$

Here dimensionless numbers are given as

$$
\begin{aligned}
& \epsilon:=\frac{\phi \alpha_{1}}{\rho K}, \gamma:=\frac{\alpha_{1}}{\rho L^{2}}, \lambda:=\frac{\psi L^{2}}{K}, H a:=\frac{\sigma B_{0}^{2} L^{2}}{\mu}, \operatorname{Pr}:=\frac{\nu}{\alpha_{3}}, S c:=\frac{\nu}{D_{B}}, \\
& \lambda_{1}:=\frac{g L^{2} \beta_{1} \theta_{0}}{A \nu}, \lambda_{2}:=\frac{g L^{2} \beta_{2} \theta_{0}^{2}}{A \nu}, N b:=\frac{\tau \phi_{0} D_{B}}{\nu}, \delta_{1}:=\frac{q_{\theta} L}{\theta_{0} k}, \delta_{2}:=\frac{q_{\phi} L}{\phi_{0} D_{B}}, \\
& N t:=\frac{\tau D_{\theta}}{\nu}, \delta_{3}:=\frac{\tau_{1}^{\alpha} \nu^{\alpha}}{\Gamma(1+\alpha) L^{2 \alpha}}, \delta_{4}:=\frac{\tau_{2}^{\beta} \nu^{\beta}}{\Gamma(1+\beta) L^{2 \beta}} .
\end{aligned}
$$

Moreover, the dimensionless coefficients of friction, Nusselt, and mass transfer Nusselt numbers are defined by

$$
\begin{aligned}
\frac{R e C_{f}}{2} & =\left[\left(1+\gamma \frac{\partial}{\partial t}\right) \frac{\partial u}{\partial y}\right]_{y=0,1}, \\
\frac{N u_{1}}{R e} & =-\left(\frac{\partial \theta}{\partial y}\right)_{y=1} \text { and } \frac{N u_{2}}{R e^{2}}=\delta_{1} t^{2}, \\
\frac{S h_{1}}{R e} & =-\left(\frac{\partial \phi}{\partial y}\right)_{y=1} \text { and } \frac{S h_{2}}{R e^{2}}=\delta_{2} t^{2} .
\end{aligned}
$$

\section{Numerical Discretization Scheme}

Numerical discretization of the flow problems (26) and (27) is presented here. The finite difference scheme(FDS) is used to discretize the fractional time derivative as given by $[15,21,43]$, while the derivative with respect to space variable is discretized with the finite element scheme(FES) proposed in $[15,21]$. The well-posedness of the flow problem can be checked in appropriate spaces [43]. Precisely, we note no ill-posedness in the defined flow problem.

Functional spaces are incorporated in the discretization of the model (26) and (27).
We point out that $\mathscr{L}^{2}(\Omega)$, square-integrable space of functions on $\Omega=(0,1)$ along with $\mathscr{L}^{2}$, norm and inner space product. Further, $\mathscr{H}^{p}(\Omega)$ stands for the Sobolev space, with $p>0, \mathscr{H}_{0}^{p}(\Omega)$ is defined as the closure of $\mathscr{C}_{0}^{\infty}(\bar{\Omega})$ in $\mathscr{H}^{p}(\Omega)$ and $\mathscr{C}_{0}^{\infty}(\bar{\Omega})$ represents infinite differentiable continuous functions, with proper compact support, in $\Omega$ [44]. Also, we use the space

$$
\mathscr{H}_{1}^{p}(\Omega)=\left\{\mathbf{u} \in \mathscr{H}^{p}(\Omega)|\mathbf{u}|_{y=1}=0\right\},
$$

with $\mathbb{L}^{2}(\Omega)=\mathscr{L}^{2}(\Omega) \times \mathscr{L}^{2}(\Omega) \times \mathscr{L}^{2}(\Omega)$ and $\mathbb{U}(\Omega)=$ $\mathscr{H}_{0}^{p}(\Omega) \times \mathscr{H}_{1}^{p}(\Omega) \times \mathscr{H}_{1}^{p}(\Omega)$.

Let $\mathscr{L}^{2}(0, T ; \mathscr{V}(\Omega)):\left[0, t_{f}\right] \longrightarrow \mathscr{V}$ equipped with

$$
\begin{aligned}
(u, v)_{\mathscr{L}^{2}\left(0, t_{f} ; \mathscr{V}(\Omega)\right)} & :=\int_{0}^{t_{f}}(u, v)_{\mathscr{V}(\Omega)} \mathrm{d} t \text { and }\|u\|_{\mathscr{L}^{2}(0, T ; \mathscr{V}(\Omega))} \\
& :=\left(\int_{0}^{t_{f}}\|u\|_{\mathscr{V}(\Omega)}^{2} \mathrm{~d} t\right)^{1 / 2} .
\end{aligned}
$$

Furthermore, $\mathscr{C}^{0}\left(\left[0, t_{f}\right] ; \mathscr{V}(\Omega)\right)$ is the space of continuous functions $u:\left[0, t_{f}\right] \longrightarrow \mathscr{V}$ with

$$
\|u\|_{\mathscr{C}^{0}\left(\left[0, t_{f}\right] ; \mathscr{V}(\Omega)\right)}:=\max _{t \in\left[0, t_{f}\right]}\|u\|_{\mathscr{V}} .
$$

Analogously, for $k \in \mathbb{N}$,

$$
\mathscr{C}^{k}\left(\left[0, t_{f}\right] ; \mathscr{V}(\Omega)\right):=\left\{u \in \mathscr{C}^{0}\left(\left[0, t_{f}\right] ; \mathscr{V}(\Omega)\right) \partial_{t}^{j} u \in \mathscr{C}^{0}([0, T] ; \mathscr{V}(\Omega)), \forall j \leq k: j \in \mathbb{N}\right\}
$$


and

$$
\|u\|_{\mathscr{C}^{k}}\left(\left[0, t_{f}\right] ; \mathscr{V}(\Omega)\right):=\max _{j=0}^{k}\left(\left\|\partial_{t}^{j} u\right\|_{\mathscr{C}^{0}\left(\left[0, t_{f}\right] ; \mathscr{V}(\Omega)\right)}\right),
$$

and also, we denote by $\mathbb{C}^{k}\left(\left[0, t_{f}\right] ; \mathbb{V}(\Omega)\right)=\left[\mathscr{C}^{k}\left(\left[0, t_{f}\right]\right.\right.$; $\mathscr{V}(\Omega))]^{3}$.

3.1. Approximations using FDS. Fractional time derivative in (26) and (27) is discretized by FDS. The interval of time, $\left[0, t_{f}\right]$ is partitioned by time step $\tau:=t_{f} / m$ so that $t_{k}:=k \tau$, and $k=0,1,2, \ldots, m$. Estimation of the derivative of time at some fixed, $t_{k}, 0<k<m$

$$
\frac{\partial u}{\partial t}\left(y, t_{k}\right) \simeq \frac{u\left(y, t_{k+1}\right)-u\left(y, t_{k}\right)}{\tau}, t_{k} \leq s \leq t_{k+1},
$$

here, $k=0$,

$$
\frac{\partial u}{\partial t}\left(y, t_{0}\right) \simeq \frac{u\left(y, t_{1}\right)-u\left(y, t_{0}\right)}{\tau} .
$$

Initial conditions of flow (27) give

$$
u\left(y, t_{1}\right) \simeq 0 \text { and } u\left(y, t_{0}\right) \simeq 0 .
$$

$$
\begin{aligned}
\mathscr{L}_{t}^{\alpha}[\theta]\left(t_{k+1}\right) & =\left(\frac{\partial}{\partial t}+\delta_{2} \frac{\partial^{\alpha+1}}{\partial t^{\alpha+1}}\right)[\theta]\left(t_{k+1}\right), \\
& \simeq \frac{\theta\left(t_{k+1}\right)-\theta\left(t_{k}\right)}{\tau}+C_{\alpha}\left[\theta\left(t_{k+1}\right)-2 \theta\left(t_{k}\right)+\theta\left(t_{k-1}\right)\right]+C_{\alpha}\left(\psi_{k}^{\alpha}[\theta]-\psi_{k-1}^{\alpha}[\theta]\right),
\end{aligned}
$$

and

$$
\begin{aligned}
\mathcal{Q}_{t}^{\alpha}[\theta]\left(t_{k+1}\right) & =\left(1+\delta_{2} \frac{\partial^{\alpha}}{\partial t^{\alpha}}\right)[\theta]\left(t_{k+1}\right) \\
& \simeq \theta\left(t_{k+1}\right)+\delta_{2} C_{\alpha}\left[\theta\left(t_{k+1}\right)-\theta\left(t_{k}\right)\right]+\delta_{2} C_{\alpha} \psi_{k}^{\alpha}[\theta]
\end{aligned}
$$

with $c_{\alpha}=\tau^{-\alpha} / \Gamma(2-\alpha)$ and where $b_{s}^{\alpha}:=(s+1)^{1-\alpha}-(s)^{1-\alpha}$, for $0 \leq s \leq m$, with

$$
\psi_{k}^{\alpha}[\theta]:=\sum_{s=1}^{k} b_{s}^{\alpha}\left[\theta\left(y, t_{k+1-s}\right)-\theta\left(y, t_{k-s}\right)\right] \text { with } \psi_{0}^{\alpha}[\theta]:=0 .
$$

3.2. Approximations using FES. In this section, the FES discretization of space variables is discussed in detail. It can be achieved by the partition of domain $\Omega=[0,1]$ with $n$
Fractional derivative of time, $\partial_{t}^{\alpha}(0<\alpha<1), 0 \leq k<m$, estimated with the Caputo derivative [43].

$$
\begin{array}{r}
\partial_{t}^{\alpha} \phi(t):=\frac{1}{\Gamma(m-\alpha)} \int_{0}^{t}(t-\tau)^{m-\alpha-1} \frac{\partial^{m}}{\partial \tau^{m}} \phi(\tau) \mathrm{d} \tau, \\
m-1<\Re e\{\alpha\}<m, m \in \mathbb{N},
\end{array}
$$

and the Gamma function $\Gamma(\cdot)$ is given as

$$
\Gamma(z):=\int_{\mathbb{R}} \xi^{z-1} e^{-\xi} \mathrm{d} \xi, z \in \mathbb{C}, \mathfrak{R}\{z\}>0 .
$$

Fractional derivative of time $\partial_{t}^{\alpha} \phi$ agrees with ordinary derivative $\partial_{t}^{m} \phi$ as $\alpha \longrightarrow m, m-1<\alpha<m$, for an integer $m$. Also, we specify the following operators

$$
\begin{aligned}
& \mathscr{L}_{t}^{\alpha}[\theta(\cdot)](t):=\left(\frac{\partial}{\partial t}+\delta_{2} \frac{\partial^{(\alpha+1)}}{\partial t^{(\alpha+1)}}\right)[\theta(t)], \\
& \widehat{Q}_{t}^{\alpha}[\theta(\cdot)](t):=\left(1+\delta_{2} \frac{\partial^{\alpha}}{\partial t^{\alpha}}\right)[\theta(t)] .
\end{aligned}
$$

Estimations with FDS of operators $\mathscr{L}_{t}^{\alpha}[\theta], Q_{t}^{\alpha}[\theta]$ can be taken as (please see [26]) 


$$
\left\{\begin{array}{l}
(1+\epsilon) \frac{\partial}{\partial t}(u, v)+\left(1+\gamma \frac{\partial}{\partial t}\right)\langle u, v\rangle+(\lambda+H a)(u, v)-\left(\left(\lambda_{1}+\lambda_{2} \theta\right) \theta, v\right)=0 \\
\operatorname{Pr} \mathscr{L}_{t}^{\alpha}(\theta, \zeta)+\langle\theta, \zeta\rangle-\operatorname{Pr} Q_{t}^{\alpha}(\theta, \zeta)\left(N b\left(\frac{\partial \theta}{\partial y} \frac{\partial \phi}{\partial y}, \zeta\right)+N t\left(\left(\frac{\partial \theta}{\partial y}\right)^{2}, \zeta\right)\right)=\left(-\delta_{1} t, \zeta(0)\right) \\
\operatorname{Sc}_{t}^{\beta}(\phi, \psi)+\langle\phi, \psi\rangle+\left(\frac{N t}{N b}\right)\langle\theta, \psi\rangle=\left(-\delta_{1} t-\delta_{2} t, \psi(0)\right) \\
u(y, 0)=, \theta(y, 0)=0=\frac{\partial \theta}{\partial t}(y, 0), \text { and } \phi(y, 0)=0=\frac{\partial \phi}{\partial t}(y, 0)
\end{array}\right.
$$

for all $(\nu, \zeta, \psi) \in \mathbb{H}(\Omega)$.

Weak form (46) is used to incorporate, discrete weak form, at $t=t_{k}, 0<k<m$

$$
\left\{\begin{array}{l}
\text { Find }\left(u_{h}\left(\cdot, t_{k+1}\right), \theta\left(\cdot, t_{k+1}\right), \phi\left(\cdot, t_{k+1}\right)\right) \in \mathbb{V}^{h}(\Omega) \operatorname{s.t.} \forall(v, \varsigma, \phi) \in \mathbb{V}^{h}(\Omega) \\
(1+\epsilon) \frac{\partial}{\partial t}\left(u_{h}\left(y, t_{k+1}\right), v\right)+\left(1+\gamma \frac{\partial}{\partial t}\right)\left\langle u_{h}\left(\left(y, t_{k+1}\right)\right), v\right\rangle+(\lambda+H a)\left(u_{h}\left(y, t_{k+1}\right), v\right) \\
-\left(\left(\lambda_{1}+\lambda_{2} \theta_{h}\left(y, t_{k+1}\right)\right) \theta_{h}\left(y, t_{k+1}\right), v\right)=0 \\
\operatorname{Pr} \mathscr{L}_{t}^{\alpha}\left(\theta_{h}\left(y, t_{k+1}\right), \zeta\right)+\left\langle\theta_{h}\left(y, t_{k+1}\right), \zeta\right\rangle-\operatorname{Pr} N b Q_{t}^{\alpha}\left(\frac{\partial \theta_{h}\left(y, t_{k+1}\right)}{\partial y} \frac{\partial \phi_{h}\left(y, t_{k+1}\right)}{\partial y}, \zeta\right) \\
-\operatorname{Pr} N t Q_{t}^{\alpha}\left(\left(\frac{\partial \theta_{h}\left(y, t_{k+1}\right)}{\partial y}\right)^{2}, \zeta\right)=\left(-\delta_{1} t, \zeta(0)\right) \\
\operatorname{Sc} \mathscr{L}_{t}^{\beta}\left(\phi_{h}\left(y, t_{k+1}\right), \psi\right)+\left\langle\phi_{h}\left(y, t_{k+1}\right), \psi\right\rangle+\frac{N t}{N b}\left\langle\theta_{h}\left(y, t_{k+1}\right), \psi\right\rangle=\left(-\delta_{1} t-\delta_{2} t, \psi(0)\right) \\
u_{h}^{0}(y)=0, \theta_{h}^{0}(y)=0=\theta_{h}^{1}(y), \phi_{h}^{0}(y)=0=\phi_{h}^{1}(y)
\end{array}\right.
$$

where $u_{h}^{0}(\cdot)=u_{h}\left(\cdot, t_{0}\right), \quad \theta_{h}^{1}(\cdot)=\theta_{h}\left(\cdot, t_{1}\right), \quad \phi_{h}^{1}(\cdot)=\phi_{h}\left(\cdot, t_{1}\right)$, $\theta_{h}^{0}(\cdot)=\theta\left(\cdot, t_{0}\right)$, and $\phi_{h}^{0}(\cdot)=\phi\left(\cdot, t_{0}\right)$. The numerical solution $\left(u_{h}, \theta_{h}, \phi_{h}\right)$ to $(47)$ is given as 


$$
\begin{aligned}
& u_{h}\left(y, t_{k+1}\right)=\sum_{p=1}^{N_{h}} u_{p}\left(t_{k+1}\right) W_{0 h}^{p}(y), y \in \bar{\Omega}, \\
& \theta_{h}\left(y, t_{k+1}\right)=\sum_{l=1}^{N_{h}} \theta_{l}\left(t_{k+1}\right) W_{1 h}^{l}(y), y \in \bar{\Omega}, \\
& \phi_{h}\left(y, t_{k+1}\right)=\sum_{l=1}^{N_{h}} \phi_{l}\left(t_{k+1}\right) W_{1 h}^{l}(y), y \in \bar{\Omega},
\end{aligned}
$$

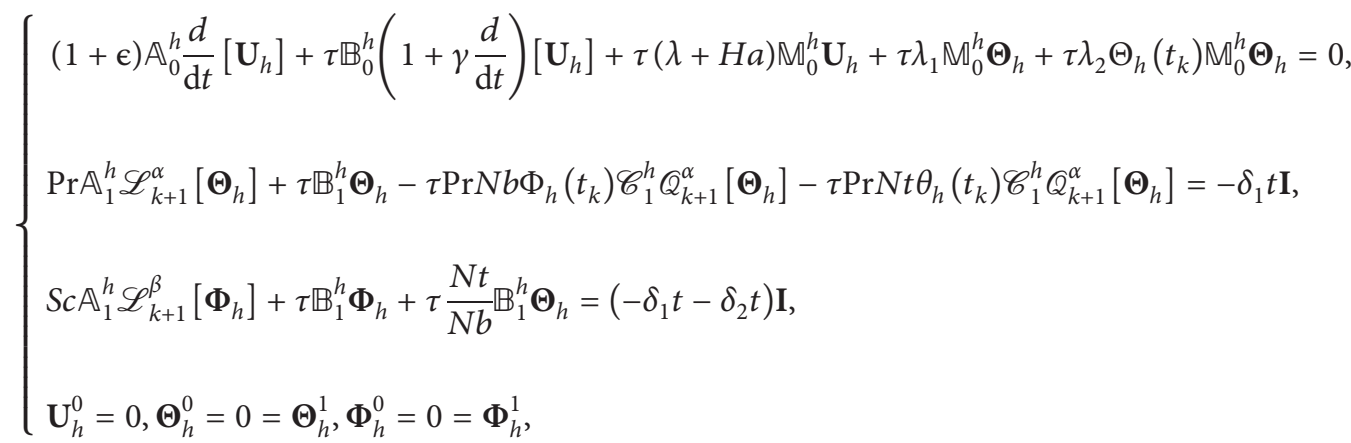

where for all $p, q=1,2, \ldots, N_{0 h}$ and $l, m=1,2, \ldots, N_{1 h}$.

$$
\begin{aligned}
\left(\mathbf{U}_{h}\right)_{p}:=u_{p},\left(\boldsymbol{\Theta}_{h}\right)_{l}:=\theta_{l},\left(\boldsymbol{\Phi}_{h}\right)_{l}:=\phi_{l}, \\
\left(\mathbb{A}_{0}^{h}\right)_{q p}:=\left(W_{0 h}^{p}, W_{0 h}^{q}\right),\left(\mathbb{B}_{0}^{h}\right)_{q p}:=\left\langle W_{0 h}^{p}, W_{0 h}^{q}\right\rangle,\left(\mathbb{M}_{0}^{h}\right)_{q p}:=\left(W_{0 h}^{p}, W_{0 h}^{q}\right), \\
\left(\mathbb{A}_{1}^{h}\right)_{l m}:=\left(W_{1 h}^{l}, W_{1 h}^{m}\right),\left(\mathbb{B}_{1}^{h}\right)_{l m}:=\left\langle W_{1 h}^{l}, W_{1 h}^{m}\right\rangle,\left(\mathscr{C}_{1}^{h}\right)_{l m}:=\left\langle W_{1 h}^{l}, W_{1 h}^{m}\right\rangle(\mathbf{I})_{m}:=(1,0,0, \ldots, 0)^{\dagger} .
\end{aligned}
$$

The system of algebraic equations, (50) has been computed by Newton's method. Linear Lagrange elements have been applied to get matrices in the defined system (50).

3.3. Convergence of Proposed Scheme. Here we have given the validation of the proposed numerical scheme so that one can confidently perform simulations of real scenarios. A comparison of numerical and theoretical error estimates is presented here. We postulate the proposed mathematical model will satisfy the given error estimates.

$$
\begin{gathered}
\left\|\zeta_{h}\left(t_{k}\right)-\zeta_{e x}\left(t_{k}\right)\right\|_{L_{2}(\Omega)} \leq D_{1}\left(h^{r+1}+\tau^{\alpha}\right), \\
\left\|\zeta_{h}\left(t_{k}\right)-\zeta_{e x}\left(t_{k}\right)\right\|_{H^{1}(\Omega)} \leq D_{2}\left(h^{r}+\tau^{\alpha}\right),
\end{gathered}
$$

here constants $D_{1}>0$ and $D_{2}>0$ are not dependent of $h$, step size, and $\tau$ (see [43]).

Theoretical and numerical error estimates are compared with the induction of source terms $F_{\mathrm{fab} 1}$ in the momentum equation, $F_{\text {fab2 }}$ in the energy equation, and $F_{\text {fab3 }}$ in the concentration equation of model (26) to obtain an exact fabricated solution. 


$$
\left\{\begin{array}{l}
(1+\epsilon) \frac{\partial u}{\partial t}=\left(1+\gamma \frac{\partial}{\partial t}\right)\left[\frac{\partial^{2} u}{\partial y^{2}}\right]-(\lambda+H a) u+\left(\lambda_{1}+\lambda_{2} \theta\right) \theta+F_{\mathrm{fab} 1}(y, t), \\
\operatorname{Pr} \frac{\partial}{\partial t}\left(1+\delta_{3} \frac{\partial^{\alpha}}{\partial t^{\alpha}}\right) \theta=\frac{\partial^{2} \theta}{\partial y^{2}}+\operatorname{Pr}\left(1+\delta_{3} \frac{\partial^{\alpha}}{\partial t^{\alpha}}\right)\left(N b \frac{\partial \theta}{\partial y} \frac{\partial \phi}{\partial y}+N t\left(\frac{\partial \theta}{\partial y}\right)^{2}\right)+F_{\mathrm{fab} 2}(y, t), \\
\operatorname{Sc} \frac{\partial}{\partial t}\left(1+\delta_{4} \frac{\partial^{\beta}}{\partial t^{\beta}}\right) \phi=\frac{\partial^{2} \phi}{\partial y^{2}}+\left(\frac{N t}{N b}\right) \frac{\partial^{2} \theta}{\partial y^{2}}+F_{\mathrm{fab} 3}(y, t),
\end{array}\right.
$$

with given conditions. The fabricated solutions completely satisfied the given equations, $u_{e x}(y, t), \theta_{\text {ex }}(y, t)$, and $\phi_{\mathrm{ex}}(y, t)$ along with conditions. For the validation of the proposed scheme, we have inserted $F_{\mathrm{fab} 1}, F_{\mathrm{fab} 2}$, and $F_{\mathrm{fab} 3}$ in the system (50). Error curves have been plotted and they are shown in Figures 1(a), 1(b), and 2.

Errors are presented in norms on log scales of $H^{1}(\Omega)$ and $L_{2}(\Omega)$ for different values of $h$. The slopes of error curves are nearly equal to 2 in the case of $H^{1}(\Omega)$ and 3 in case of $L_{2}(\Omega)$. Theoretically, in 3.15 and 3.16, Lagrange polynomials are of degrees $r+1$ and $r$, respectively. In the proposed scheme we have incorporated a second-degree Lagrange polynomial, so exact values must be equal to 2 in case of $H^{1}(\Omega)$ and 3 in case of $L_{2}(\Omega)$. This shows the agreement between theoretical and numerical error estimates. So, the proposed scheme (50), is convergent and can efficiently handle real simulations of proposed model (26). The error estimates for velocity, temperature, and concentration are given in Tables 1 and 2.

\section{Approximate Simulated Results}

This section deals with the results of the velocity field, temperature field, and concentration of nanofluid with nonlinear convection and flux conditions using MATLAB R2017a. Brownian phenomena of motion and thermophoresis are analyzed while evaluating this study. Fluid is flowing through the Darcy porous medium. Flow and magnetic fields are orthogonal. The proposed algorithm is employed for numerical solutions of governing nonlinear fractional equations. Discretization of time and space variables is carried out by FDS and FES, respectively. We analyze the characteristics of physical numbers on flow field generation. Transfer of heat and mass are also discussed for various pertinent numbers. Variations of flow quantities with the variation of different nondimensional numbers help to understand the problem. Simulated results are seen over intervals of time $[0,2]$, and $[0, \sqrt{2}]$. Figure 3 , is used for demonstration of velocity behavior with the variation of $0<\alpha<1$. It is observed that for higher values of $\alpha$, velocity increases (see Figure 3(a)). However, this occurrence is hereditary in character and cannot be considered to be other variations of pertinent parameters.

Change in velocity with the variation of $0.1 \leq H a \leq 3$, and $0.1 \leq \gamma \leq 0.8$ are plotted in Figure 3(b). The plot showed that a decrease in velocity is noted for higher values of $\mathrm{Ha}$ and opposite behavior is obtained for the viscoelastic parameter, $\gamma$. As $\mathrm{Ha}$ increases, Lorentz force decreases, which slows down fluid motion. As a result, velocity decreases for higher values of $\mathrm{Ha}$. Porosity parameter $0 \leq \lambda \leq 7$ and convection number $0.1 \leq \lambda_{1} \leq 3.5$ influence on the velocity, which is plotted in Figure 4. It is noted that velocity decreases for higher values of $\lambda$, while an opposite trend is noted for $\lambda_{1}$ in Figure 4(a). With the increase of $\lambda$, permeability decreases while porosity increases. So, the velocity profile decreases with the increase of $\lambda$. Force of inertia is inversely related to $\lambda_{1}$ and direct influence is given for buoyancy force. As $\lambda_{1}>0$, heat transfers from plates to fluid. So, $\left(\theta_{s_{1}}-\theta_{0}\right)$ and $\left(\theta_{s_{2}}-\right.$ $\theta_{0}$ ) increase. Consequently, the increase in $\lambda_{1}$ increases buoyancy forces, $\left(\theta_{s_{1}}-\theta_{0}\right)$ and $\left(\theta_{s_{2}}-\theta_{0}\right)$. So, increase in fluid velocity is noted in this case.

Similar behavior of nonlinear convection number $0.1 \leq \lambda_{2} \leq 6.5$ is noted in Figure 4(b). When there is convection, it is nonlinear in nature, so as noted, the same results are observed for the convection parameter $\lambda_{1}$. It is noted that an increase in the Darcy resistance number, $0.1 \leq \epsilon \leq 6.5$ reflects a decrease in velocity. This is because an increase in $\epsilon$ permeability decreases while porosity increases. Hence, a decrease in velocity with an increase in Darcy resistance number is noted.

Figure 5 is plotted to observe the temperature profile, for several values of $\alpha$. Temperature profile, increases as $\alpha$ increases in Figure 5(a). Temperature profile behavior with the increase of pedesis number $0.1 \leq N b \leq 4$, Schmidt parameter $0.1 \leq S c \leq 4$ are observed via Figure 5(b). It is noted that temperature increases. as $\mathrm{Sc}, \mathrm{Nb}$ increase. Momentum diffusivity enhances with the increase of $S c$, which increases friction between different layers of the flow domain. As a result, an increase in temperature is seen with the increase of Sc. A decrease of temperature with the decrease of base fluid heat capacity is noted with an increase of $\mathrm{Nb}$.

The influence of $0.1 \leq \operatorname{Pr} \leq 7$, Prandtl number, $0.1 \leq N t \leq 4$ thermophoresis number, on temperature is plotted in Figure 6. With the increase of $N t$, the temperature profile increases, while the opposite trend is observed in the case of $\mathrm{Pr}$ in Figure 6(a). With the increase of $N t$, the heat capacity of the nanofluid decreases, as a result, the temperature profile increases, for higher values of $N t$. With the increase of $\mathrm{Pr}$, thermal diffusivity, decreases so temperature decreases with the increase of Pr. Figure 6(b) is plotted to show the influence of heat flux, $0.1 \leq \delta_{1} \leq 0.8$ and mass flux, $0.1 \leq \delta_{2} \leq 0.8$ numbers on temperature. It is noted 


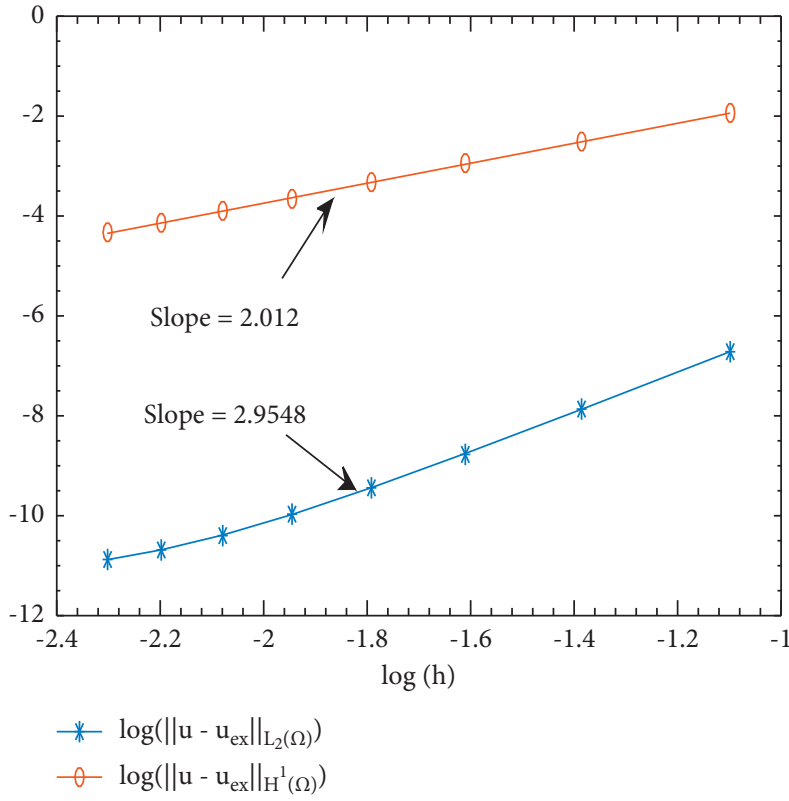

(a)

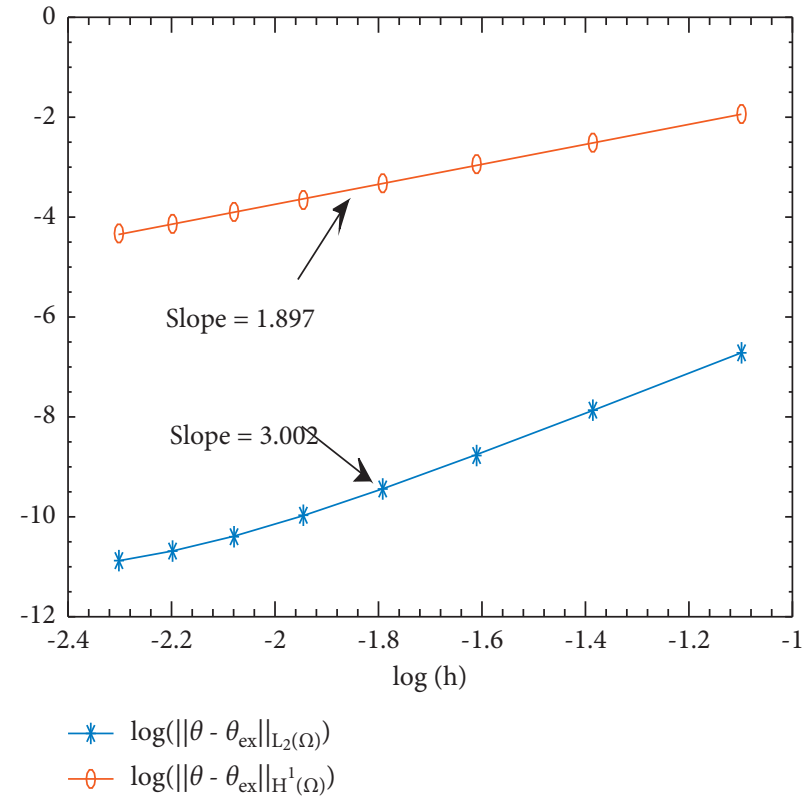

(b)

Figure 1: Error estimates for temperature and velocity. (a) Velocity $H_{1}$ and $L_{2}$ error curves. (b) Temperature $H_{1}$ and $L_{2}$ error curves.

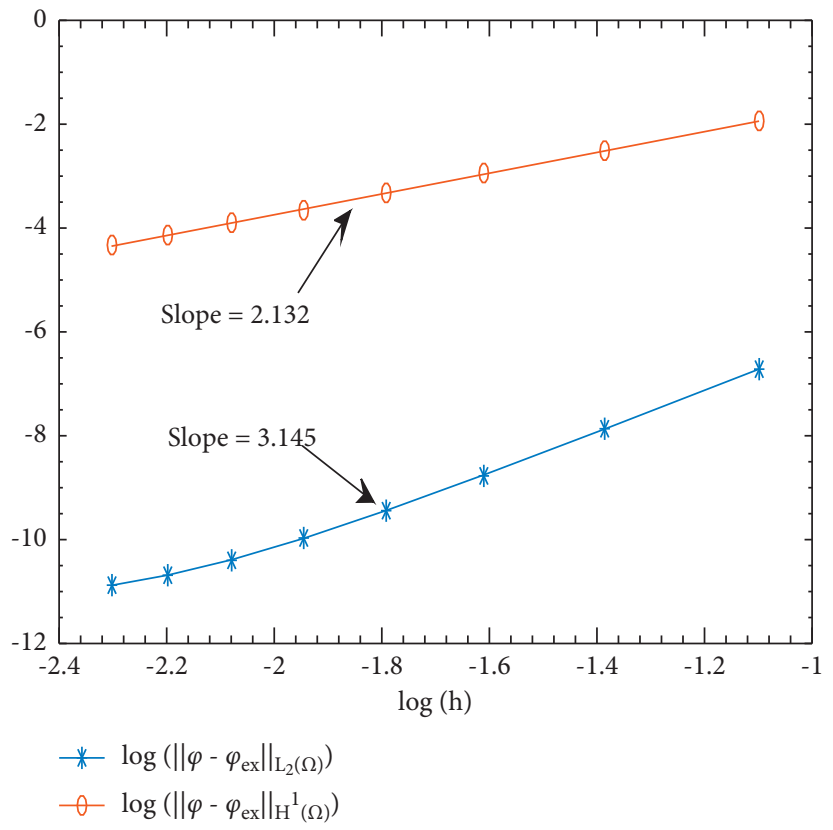

FIGURE 2: Concentration $H_{1}$ and $L_{2}$ error curves.

TABLE 1: Error estimates for velocity and temperature.

\begin{tabular}{lccccc}
\hline$n$ & $h$ & \multicolumn{2}{c}{ Error estimates of velocity } & \multicolumn{2}{c}{ Error estimates of temperature } \\
& & $\left\|u-u_{\mathrm{ex}}\right\|_{L_{2}(\Omega)}$ & $\left\|u-u_{\mathrm{ex}}\right\|_{H^{1}(\Omega)}$ & $\left\|\theta-\theta_{\text {ex }}\right\|_{L_{2}(\Omega)}$ & $\left\|\theta-\theta_{\mathrm{ex}}\right\|_{H^{1}(\Omega)}$ \\
\hline 7 & 0.3000 & $2.5816528 \times 10^{-5}$ & $1.2760729 \times 10^{-1}$ & $1.211254 \times 10^{-4}$ & $1.4354533 \times 10^{-1}$ \\
10 & 0.2360 & $1.7530926 \times 10^{-5}$ & $1.0335894 \times 10^{-1}$ & $3.809745 \times 10^{-5}$ & $8.0745776 \times 10^{-2}$ \\
13 & 0.1807 & $1.2588759 \times 10^{-5}$ & $8.5420742 \times 10^{-2}$ & $1.584725 \times 10^{-5}$ & $5.1678524 \times 10^{-2}$ \\
16 & 0.1728 & $9.5167115 \times 10^{-6}$ & $7.1777154 \times 10^{-2}$ & $7.961548 \times 10^{-6}$ & $3.5888249 \times 10^{-2}$ \\
19 & 0.1529 & $7.5460756 \times 10^{-6}$ & $6.1157856 \times 10^{-2}$ & $4.640422 \times 10^{-6}$ & $2.6367821 \times 10^{-2}$ \\
22 & 0.1502 & $6.2508125 \times 10^{-6}$ & $5.2734257 \times 10^{-2}$ & $3.080778 \times 10^{-6}$ & $2.0187853 \times 10^{-2}$ \\
25 & 0.1456 & $5.3821874 \times 10^{-6}$ & $4.5938245 \times 10^{-2}$ & $2.2959072 \times 10^{-6}$ & $1.5949251 \times 10^{-2}$ \\
28 & 0.1378 & $4.7890743 \times 10^{-6}$ & $4.0375489 \times 10^{-2}$ & $1.8817548 \times 10^{-6}$ & $1.2918457 \times 10^{-2}$ \\
\hline
\end{tabular}


TABLE 2: Error estimates for concentration.

\begin{tabular}{lccr}
\hline$n$ & $h$ & \multicolumn{2}{c}{ Error estimates of concentration } \\
& & $\left\|u-u_{\text {ex }}\right\|_{L_{2}(\Omega)}$ & \multicolumn{1}{c}{$\| .2760729 \times 10^{-2}$} \\
7 & 0.3000 & $3.6875492 \times 10^{-4}$ & $1.0335894 \times 10^{-2}$ \\
10 & 0.2360 & $1.7254876 \times 10^{-4}$ & $9.5124783 \times 10^{-3}$ \\
13 & 0.1807 & $1.4258149 \times 10^{-4}$ & $8.2549678 \times 10^{-3}$ \\
16 & 0.1728 & $8.1568473 \times 10^{-5}$ & $6.3548962 \times 10^{-3}$ \\
19 & 0.1529 & $6.4582167 \times 10^{-5}$ & $5.7854216 \times 10^{-3}$ \\
22 & 0.1502 & $4.8723459 \times 10^{-5}$ & $4.3698524 \times 10^{-3}$ \\
25 & 0.1456 & $3.1248753 \times 10^{-5}$ & $3.9645871 \times 10^{-3}$ \\
28 & 0.1378 & $2.4529751 \times 10^{-5}$ & \\
\hline
\end{tabular}

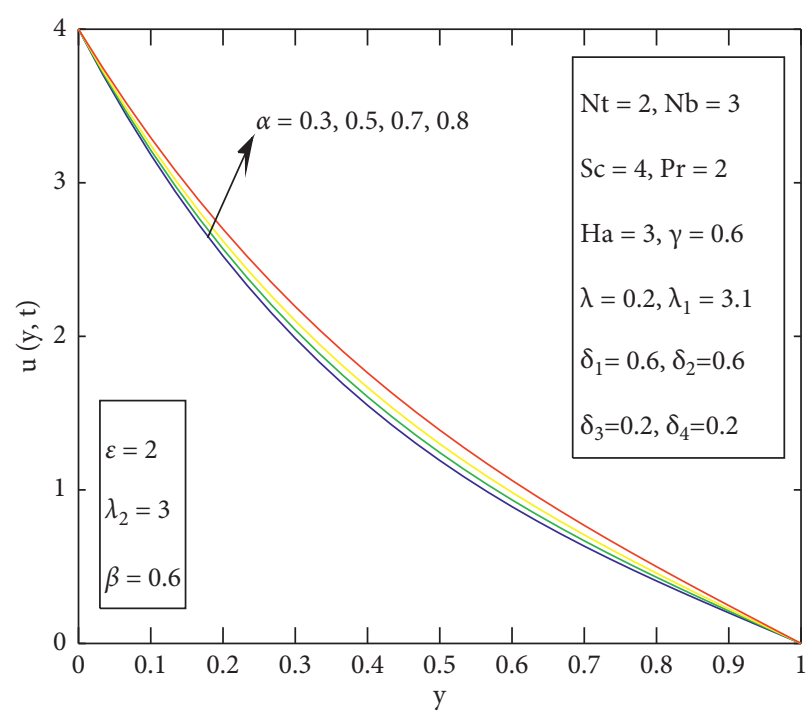

(a)

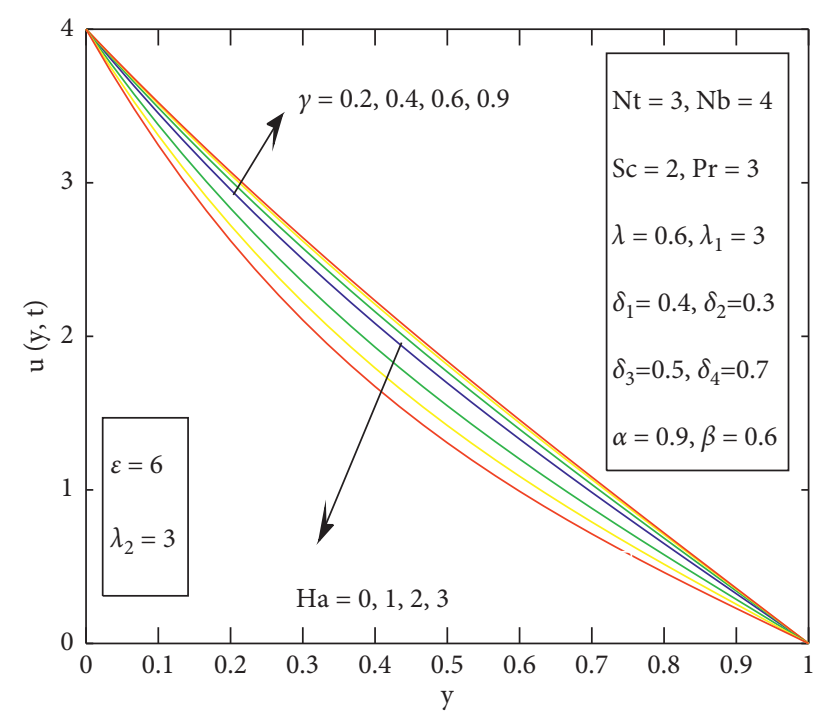

(b)

FIGURE 3: Influence of noninteger number, Hartmann number, and viscoelastic number on velocity. (a) Change in velocity with $\alpha$. (b) Change in velocity with $H_{a}$ and $\Upsilon$.

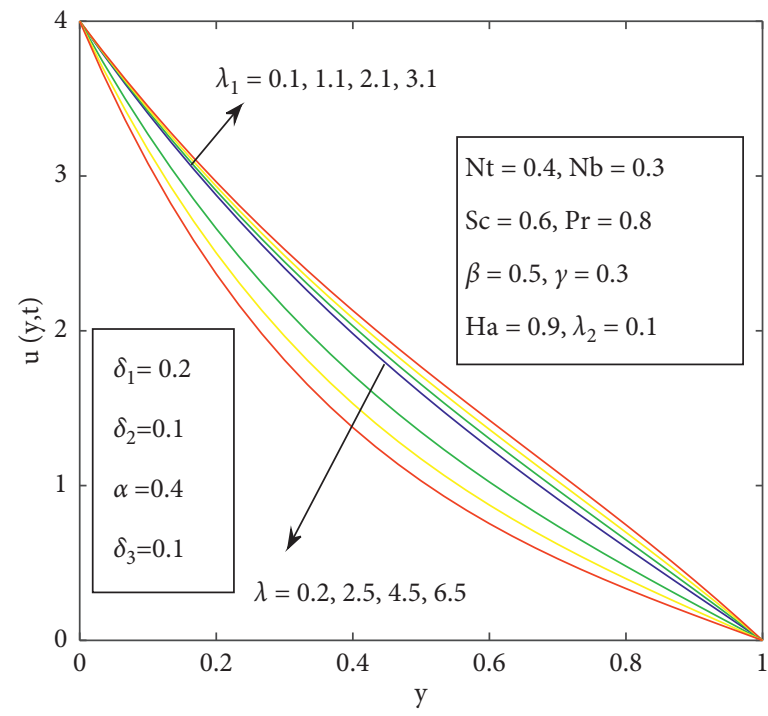

(a)

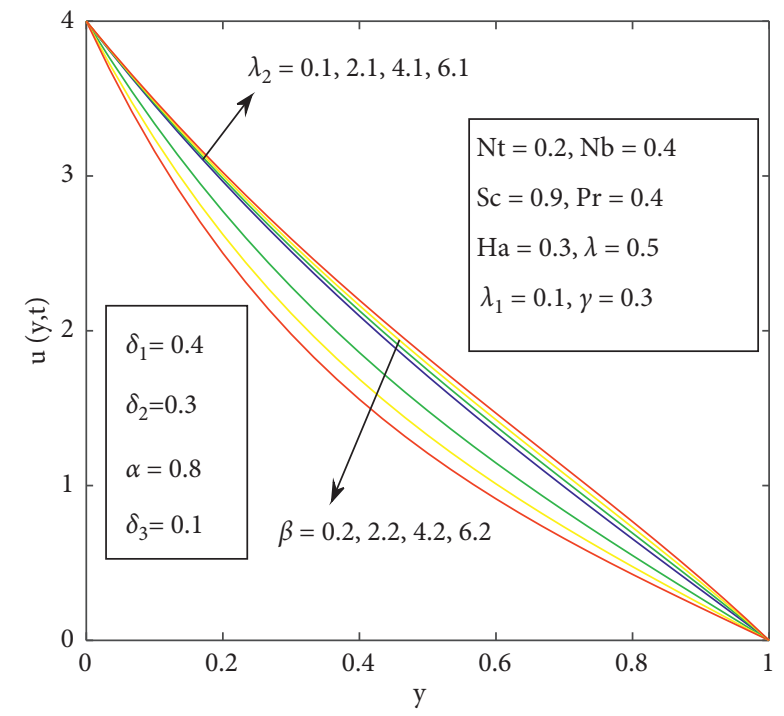

(b)

FIGURE 4: Influence of porosity, Darcy resistance, and convection numbers on velocity. (a) Change in velocity with $\lambda$ and $\lambda_{1}$. (b) Change in velocity with $\lambda_{2}$ and $\varepsilon$. 


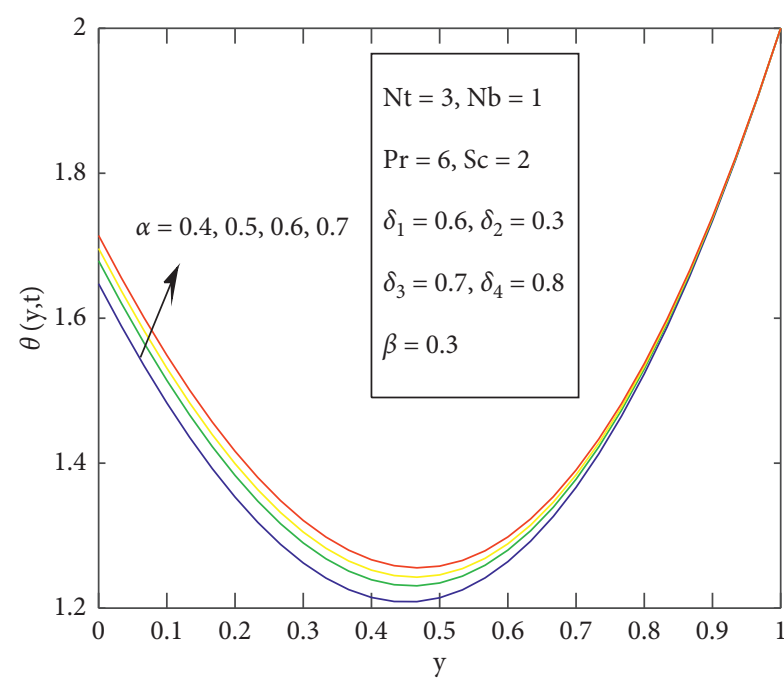

(a)

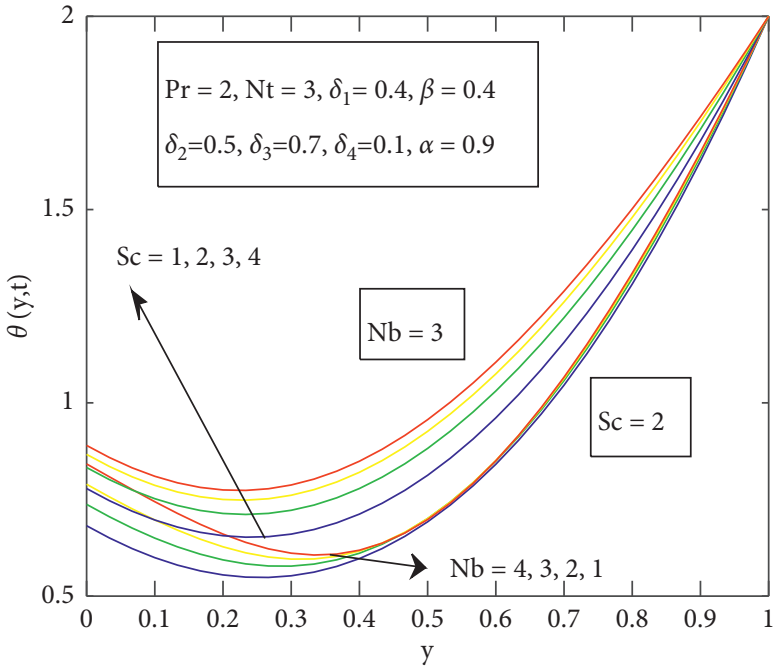

(b)

FIGURE 5: Influence of noninteger number, pedesis parameter, and Schmidt number on temperature. (a) Change in temperature with $\alpha$. (b) Change in temperature with $S c$ and $N b$.

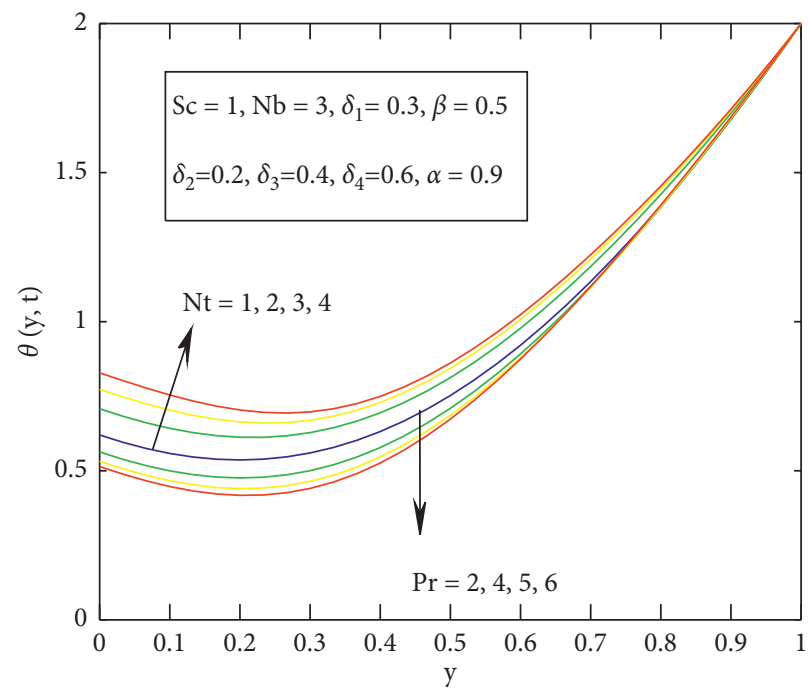

(a)

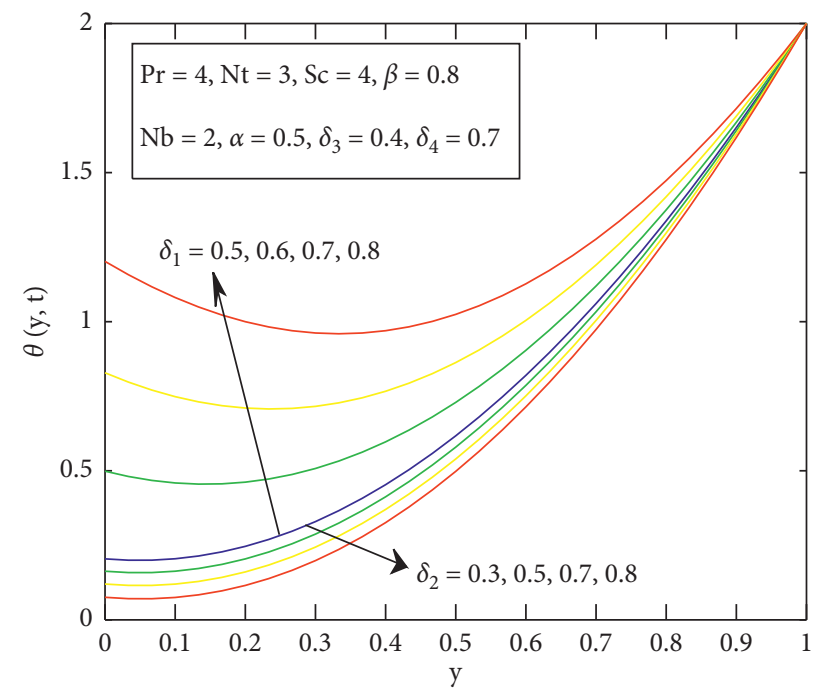

(b)

FIGURE 6: Influence of Prandtl number, heat and mass fluxes, and thermophoresis numbers on temperature. (a) Change in temperature with $\mathrm{Pr}$ and Nt. (b) Change in temperature with $\delta_{1}$ and $\delta_{2}$.

temperature increases for higher values of $\delta_{1}$ while opposite trends are seen for the case of $\delta_{2}$. With the increase of $\delta_{1}$, thermal conductivity, the fluid decreases which decreases the heat passage rate through the base fluid, so temperature increases for higher values of $\delta_{1}$.

Figure 7 is outlined to observe concentration change for various values of $\alpha$. Concentration is at a higher level for higher values of $\alpha$ in Figure 7(a). Effects of thermophoresis, $\mathrm{Nt}$, pedesis, $\mathrm{Nb}$ numbers on concentration are plotted in Figure 7(b). Concentration increases, for higher values of $N t$, while the opposite behavior is noted for $\mathrm{Nb}$. An increase in concentration, is because of an increase in the coefficient of thermophoretic diffusion.
The influence of Prandtl $P r$, and Schmidt Sc numbers on concentration can be seen via Figure 8. Concentration increases for higher values of $\mathrm{Pr}$, while the opposite behavior is noted in the case of $S c$ as shown in Figure 8(a). Momentum diffusivity increases for higher values of $\mathrm{Pr}$, as a result, concentration is at a greater level for higher values of $\mathrm{Pr}$. The viscosity of fluid increases, and the Brownian diffusion coefficient decreases for higher values of Sc. So, the concentration remains at a lower level for higher values of $S c$. Figure 8(b) is outlined to observe the impact of heat flux $\delta_{1}$, and mass flux $\delta_{2}$ numbers on concentration. Concentration increases for higher values of $\delta_{1}$ and $\delta_{2}$. The rate of transfer of concentration decreases for higher values of $\delta_{2}$. So, the 


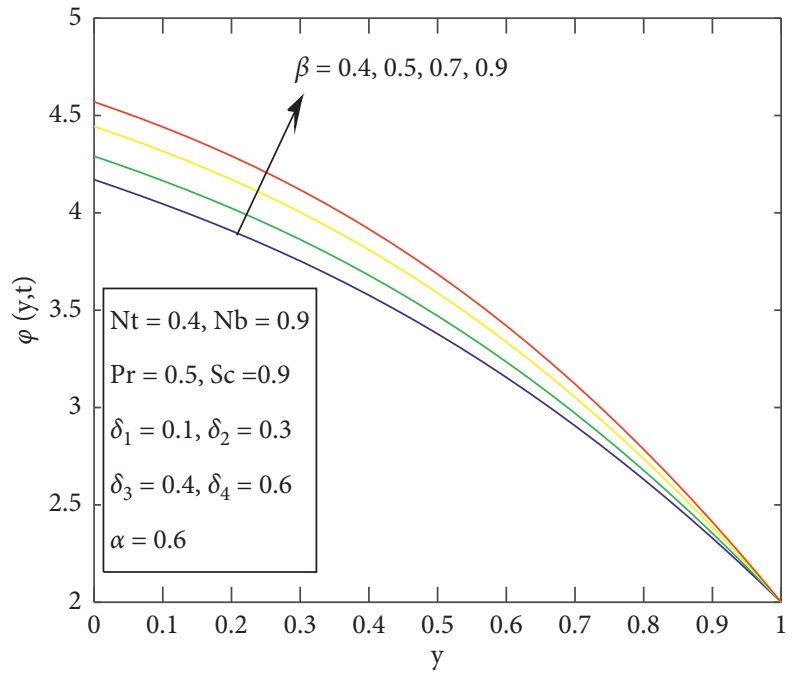

(a)

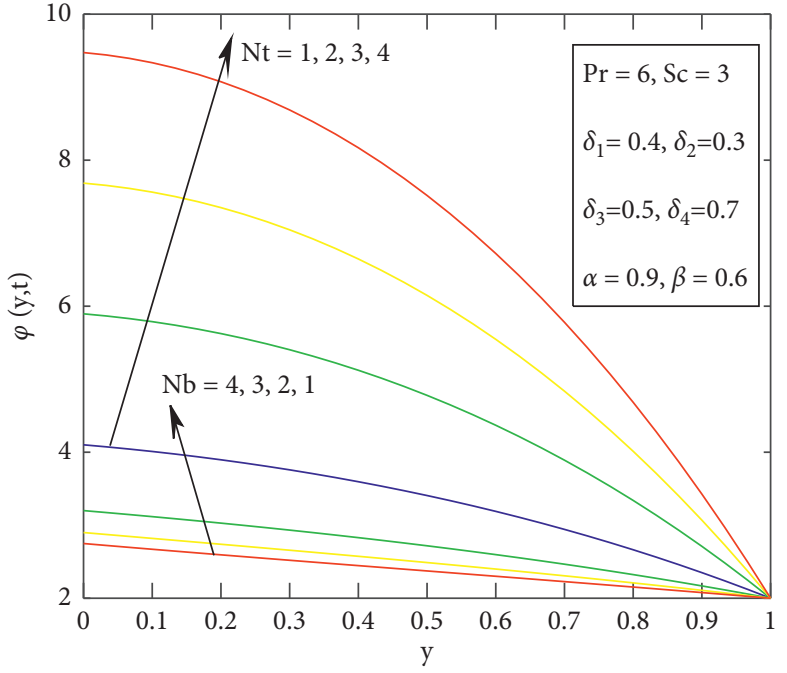

(b)

FiguRE 7: Influence of fractional number, thermophoresis, and pedesis numbers on concentration. (a) Change in concentration with $\alpha$. (b) Change in concentration with $\mathrm{Nt}$, and $\mathrm{Nb}$.

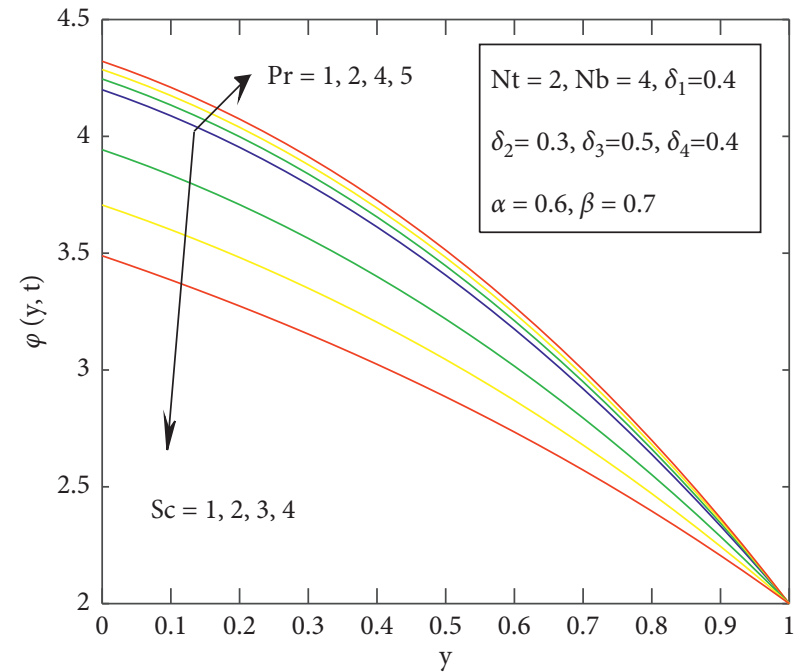

(a)

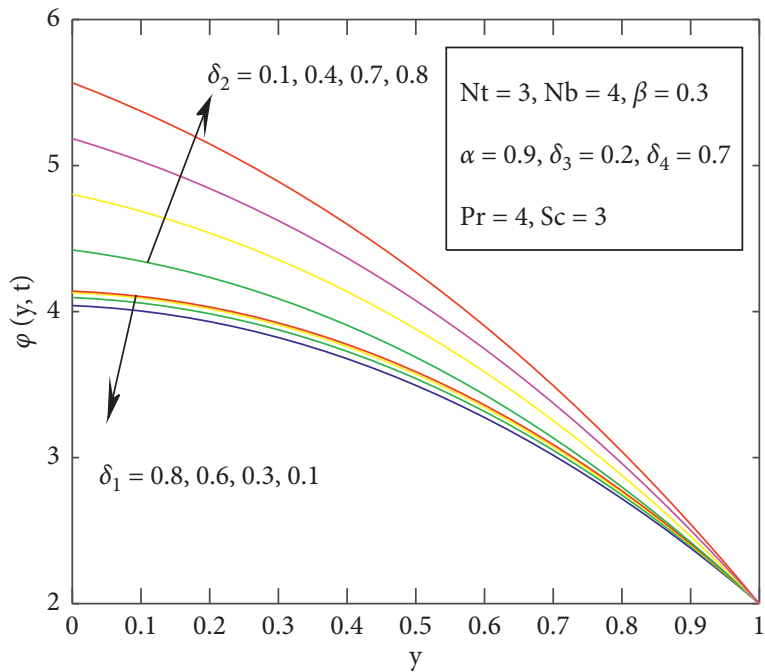

(b)

Figure 8: Influence of Prandtl number, heat and mass fluxes numbers, and Schmidt number on concentration. (a) Change in concentration with $P r$, and $S c$. (b) Change in concentration with $\delta_{1}$, and $\delta_{2}$.

concentration increases for higher values of $\delta_{2}$. At the end Figures 9(a) and 9(b)-12(a) and 12(b) are plotted for time dependent velocity, temperature, and concentration profiles. These figures showed anomalous character of noninteger nanofluid.
Variations of skin friction with pertinent fractional model parameters are examined via Tables 3 and 4. Skin friction magnitude increases with the increase of $\alpha, \beta, \lambda, \delta_{2}$, $\mathrm{Pr}$, and $\mathrm{Ha}$, while it decreases with the increase of $\gamma, \lambda_{1}, \lambda_{2}$, $\delta_{1}, S c, N t$, and $N b$. 


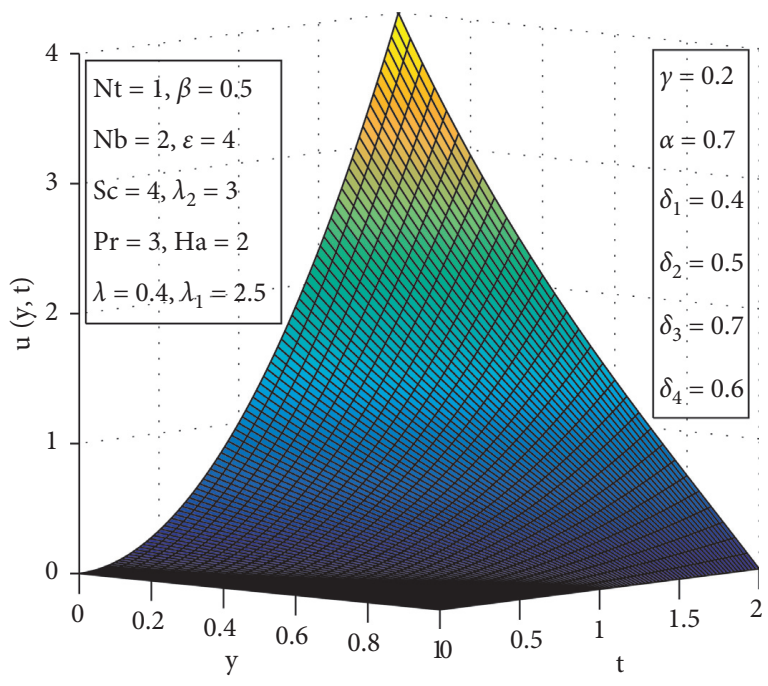

(a)

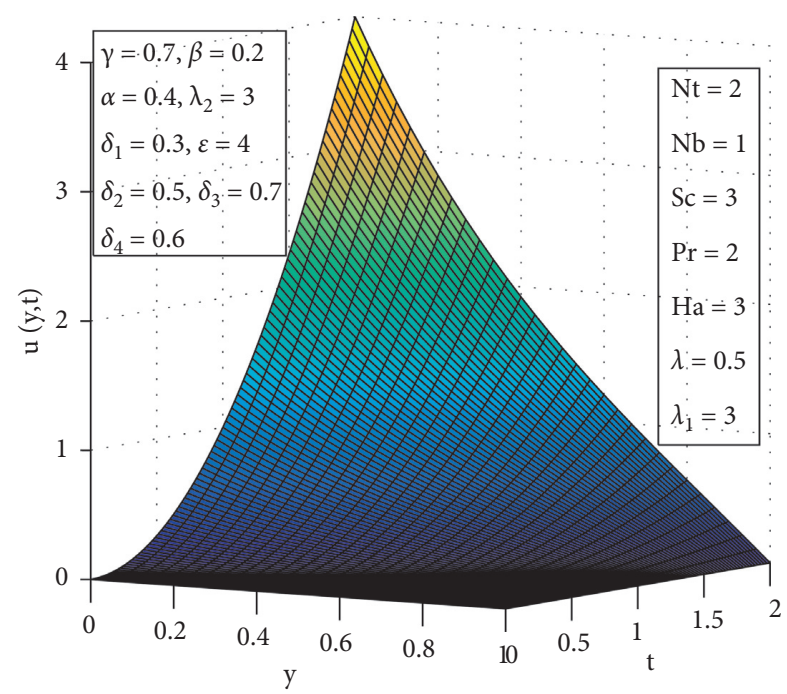

(b)

FIGURE 9: Unsteady velocity for various values of physical parameters over the interval $[0,2]$.

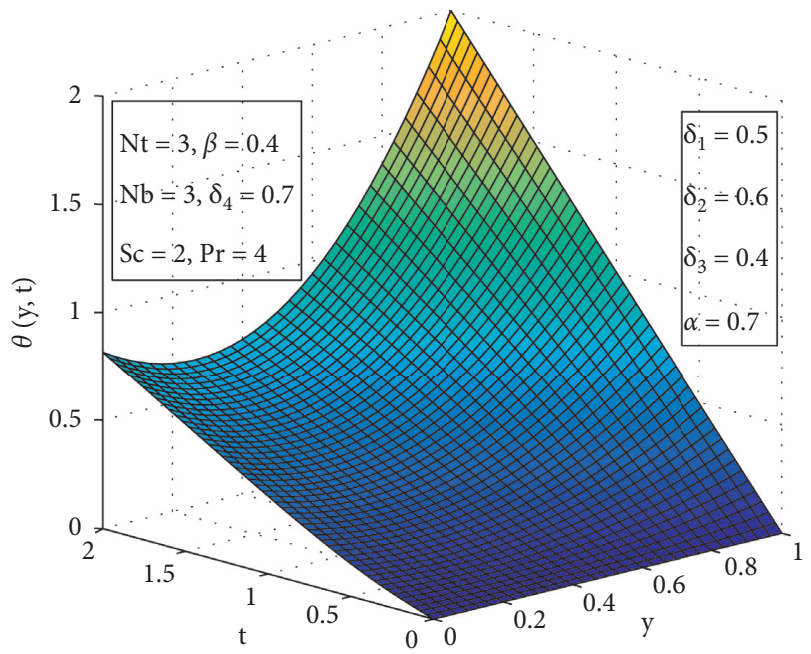

(a)

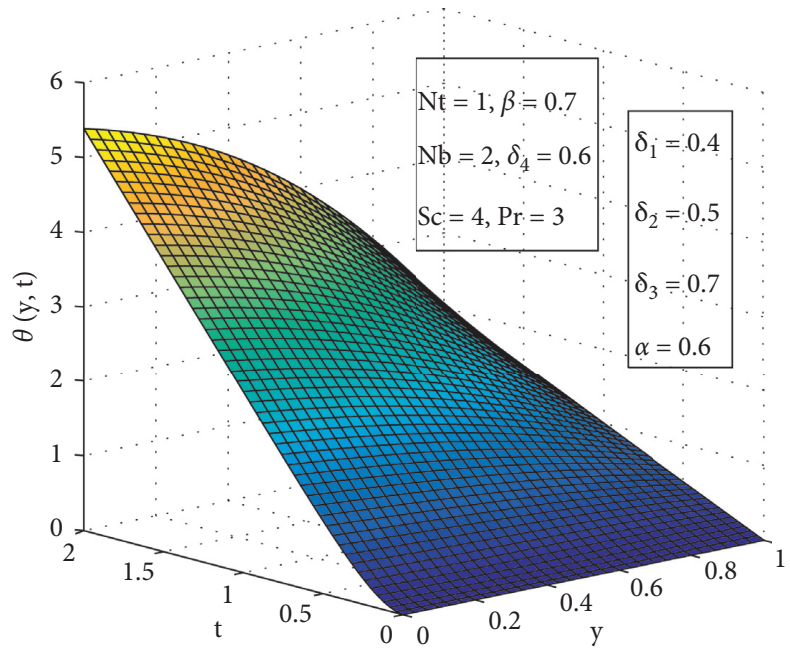

(b)

FIgURE 10: Unsteady temperature and concentration for various values of physical parameters over the interval $[0, \sqrt{2}]$. 


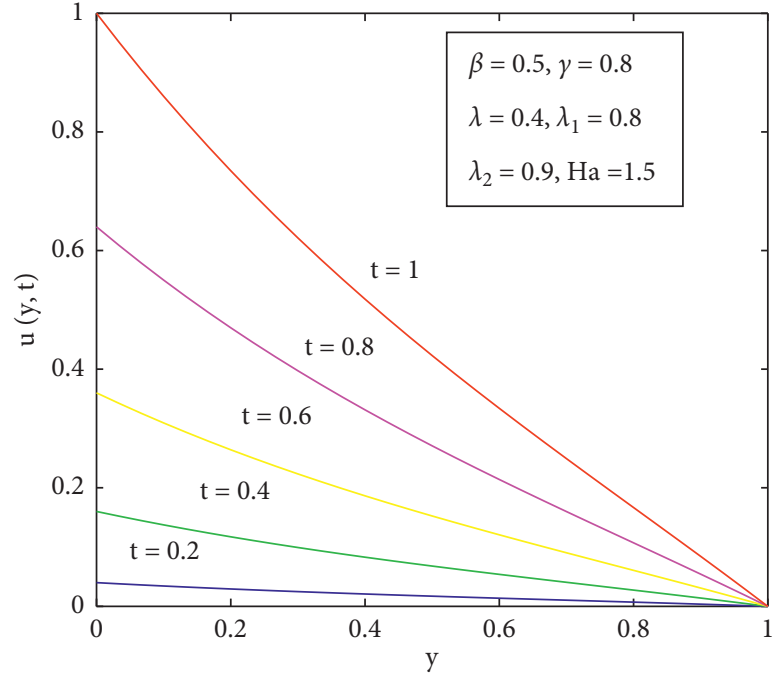

(a)

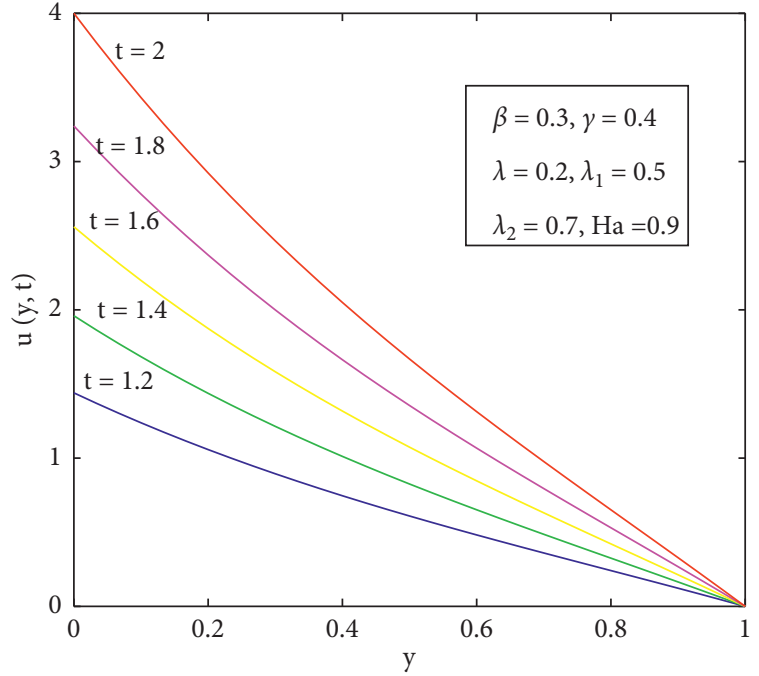

(b)

Figure 11: Velocity profiles for various final times for $N t=0.6, S c=0.5, N b=0.7, \delta_{1}=0.5, \operatorname{Pr}=0.8, \delta_{2}=0.3, \delta_{3}=0.1$, and $\delta_{4}=0.1$. (a) Variations of velocity profile when $\alpha=0.5$. (b) Variations of velocity profile when $\alpha=0.1$.

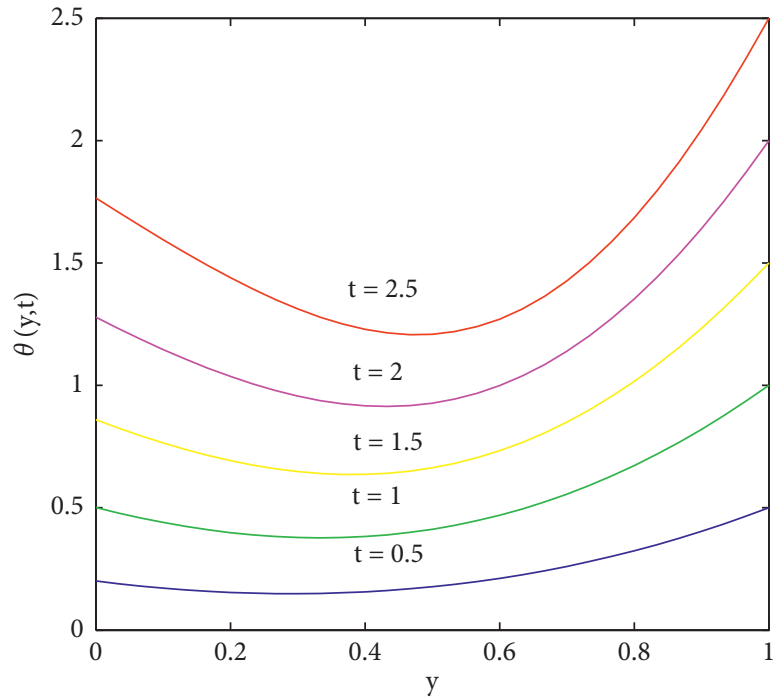

(a)

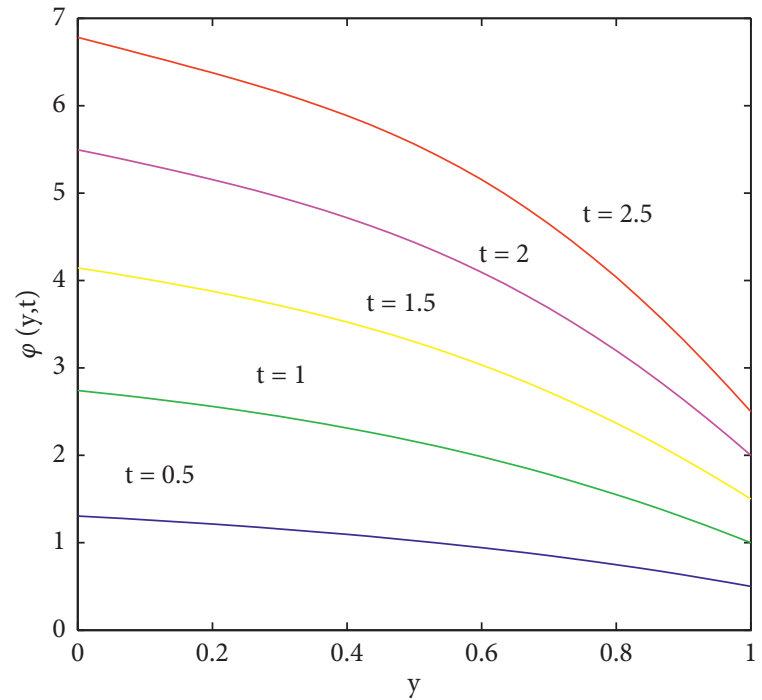

(b)

Figure 12: Temperature and concentration profiles for various final times for $N t=0.9, S c=0.3, N b=0.8, \delta_{1}=0.7, \operatorname{Pr}=1, \delta_{2}=0.8$, $\delta_{3}=0.1$ and $\delta_{4}=0.1$. (a) Variations of temperature profile when $\alpha=0.7$. (b) Variations of temperature profile when $\beta=0.7$.

TABLE 3: Influence of fractional flow numbers on the coefficient of friction at $(y, t)=(0,0.1)$ for $S c=0.5, N t=0.3, P r=0.4, N b=0.4$, $\delta_{1}=0.4, \delta_{2}=0.6, \delta_{3}=0.01, \delta_{4}=0.01, \alpha=0.5$, and $\beta=0.5$.

\begin{tabular}{|c|c|c|c|c|c|c|}
\hline$\epsilon$ & $\gamma$ & $\lambda$ & $\lambda_{1}$ & $\lambda_{2}$ & $\mathrm{Ha}$ & $\mathrm{ReC}_{f} / 2$ \\
\hline 0.1 & 0.2 & 0.1 & 0.2 & 0.1 & 0.2 & -2.351733 \\
\hline 0.2 & & & & & & -2.430800 \\
\hline \multirow[t]{5}{*}{0.3} & 0.2 & & & & & -2.507821 \\
\hline & 0.3 & & & & & -2.305209 \\
\hline & 0.4 & 0.1 & & & & -2.168051 \\
\hline & & 0.2 & & & & -2.177760 \\
\hline & & 0.3 & 0.2 & & & -2.187445 \\
\hline
\end{tabular}


TABLE 3: Continued.

\begin{tabular}{|c|c|c|c|c|c|c|}
\hline$\epsilon$ & $\gamma$ & $\lambda$ & $\lambda_{1}$ & $\lambda_{2}$ & $\mathrm{Ha}$ & $\operatorname{ReC}_{f} / 2$ \\
\hline & & & 0.3 & & & -2.133404 \\
\hline & & & 0.4 & 0.1 & & -2.079362 \\
\hline & & & & 0.2 & & -2.078998 \\
\hline & & & & 0.3 & 0.2 & -2.078633 \\
\hline & & & & & 0.3 & -2.088548 \\
\hline & & & & & 0.4 & -2.098437 \\
\hline
\end{tabular}

TABLE 4: Influence of fractional flow numbers on the coefficient of friction at $(y, t)=(0,0.1)$ for $\epsilon=0.3, \beta=0.3, \gamma=0.4, \lambda=0.1, H a=0.5$, $\delta_{3}=0.01, \delta_{4}=0.01, \lambda_{1}=0.5$, and $\lambda_{2}=0.7$.

\begin{tabular}{|c|c|c|c|c|c|c|c|}
\hline$\alpha$ & $\delta_{1}$ & $\delta_{2}$ & $\mathrm{Pr}$ & $S c$ & $N t$ & $\mathrm{Nb}$ & $\mathrm{ReC}_{f} / 2$ \\
\hline 0.1 & 0.5 & 0.4 & 0.6 & 0.5 & 0.4 & 0.5 & -0.314325 \\
\hline 0.2 & & & & & & & -0.567381 \\
\hline \multirow[t]{13}{*}{0.3} & 0.5 & & & & & & -0.936514 \\
\hline & 0.6 & & & & & & -0.890553 \\
\hline & 0.7 & 0.4 & & & & & -0.844409 \\
\hline & & 0.5 & & & & & -0.844564 \\
\hline & & 0.6 & 0.6 & & & & -0.844719 \\
\hline & & & 0.7 & & & & -0.870374 \\
\hline & & & 0.8 & 0.5 & & & -0.892903 \\
\hline & & & & 0.6 & & & -0.892822 \\
\hline & & & & 0.7 & 0.4 & & -0.892728 \\
\hline & & & & & 0.5 & & -0.892449 \\
\hline & & & & & 0.6 & 0.5 & -0.892164 \\
\hline & & & & & & 0.6 & -0.891937 \\
\hline & & & & & & 0.7 & -0.891685 \\
\hline
\end{tabular}

\section{Conclusion}

The flow of a nanofluid with a fractional derivative of time and nonlinear convection is studied in this communication. The fractional derivative of time is used to analyze the memory characteristics. Applications of Darcy porous medium can be encountered in numerous industries. Brownian motion effects are also considered while formulating the flow domain. A numerical algorithm is incorporated to estimate variations in the flow field. Flow is carried out by variable acceleration of the lower boundary. The flow direction and the applied magnetic field are orthogonal. Conditions for heat and mass gradients are considered at the bottom boundary, while quadratic variation is seen at the upper boundary. Enhancement in velocity is noted, for higher values of $\alpha, \lambda_{1}, \gamma$, and $\lambda_{2}$, and opposite behavior is seen in case of $\lambda, H a$, and $\beta$. Also, the enhancement of temperature profiles are observed for higher values of $N t, N b, S c, \alpha$, and $\delta_{1}$, and opposite behavior is noted in case of $\operatorname{Pr}$ and $\delta_{2}$. Concentration increases with the increase of $\alpha, N t, \operatorname{Pr}, \delta_{1}$, and $\delta_{2}$, and decreasing behavior is observed for $\mathrm{Nb}$ and $\mathrm{Sc}$. It is believed that fractional porous medium flows of nanofluids can be tackled with the current study. Flow, discussed in this communication, can be tackled in the manufacture of fiber and in geology. Moreover, the current study can be extended to Maxwell fluid flow. An analysis can also be performed to tackle nonlinear radiation effects in a fluid of differential type with fractional derivative.

\section{Nomenclature}

$\begin{array}{ll}u: & \text { Velocity component } \\ t: & \text { Time } \\ \phi: & \text { Fluid concentration } \\ \phi_{0}: & \text { Initial concentration } \\ c_{p}: & \text { Nanoparticles specific heat } \\ h_{p}: & \text { Nanoparticles enthalpy } \\ \alpha, \beta: & \text { Fractional derivatives } \\ \alpha_{3}: & \text { Thermal diffusivity } \\ g: & \text { Gravitational acceleration } \\ \tau: & \text { Heat capacities ratio } \\ \sigma: & \text { Electrical conductivity } \\ \psi: & \text { Porosity } \\ \alpha_{1}: & \text { Second-grade fluid material parameter } \\ k: & \text { Thermal conductivity } \\ q_{\theta}: & \text { Heat flux } \\ \epsilon: & \text { Darcy resistance parameter } \\ \lambda: & \text { Porosity parameter } \\ \lambda_{1}, \lambda_{2}: & \text { Convection parameters } \\ N t: & \text { Thermophoresis parameter } \\ \delta_{1}: & \text { Heat flux parameter } \\ \delta_{3}, \delta_{4}: & \text { Relaxation time parameters } \\ \text { Pr: } & \text { Prandtl parameter } \\ y: & \text { Space coordinate } \\ \theta: & \text { Fluid temperature } \\ \theta_{0}: & \text { Initial temperature } \\ c_{f}: & \text { Specific heat } \\ & \end{array}$




$\begin{array}{ll}\rho_{f}: & \text { Fluid density } \\ \rho_{p}: & \text { Nanoparticles density } \\ \tau_{1}, \tau_{2}: & \text { Relaxation times } \\ D_{B}: & \text { Diffusion coefficient } \\ D_{\theta}: & \text { Thermophoretic coefficient } \\ \beta_{1}, \beta_{2}: & \text { Thermal expansion coefficients } \\ K: & \text { Permeability } \\ B_{0}: & \text { Applied magnetic field } \\ A: & \text { Dimensional constant } \\ v: & \text { Kinematic viscosity } \\ q_{\phi}: & \text { Concentration flux } \\ \gamma: & \text { Viscoelastic parameter } \\ \lambda: & \text { Magnetic parameter } \\ N b: & \text { Pedesis parameter } \\ N b: & \text { Pedesis parameter } \\ \delta_{2}: & \text { Mass flux parameter } \\ S c: & \text { Schmidt parameter } \\ \text { Re: } & \text { Reynold parameter. }\end{array}$

\section{Data Availability}

No data were used to support this study.

\section{Conflicts of Interest}

The authors declare that there are no conflicts of interest in this study.

\section{Authors' Contributions}

All authors contributed equally to the commenting, proofreading, and writing of this communication.

\section{Acknowledgments}

The authors extend their appreciation to the Deanship of Scientific Research at King Khalid University, Abha, Saudi Arabia, for funding this work through the research groups program under grant number RGP.2/20/43.

\section{References}

[1] J. Julyan and A. J. Hutchinson, "Conservation laws and conserved quantities of the governing equations for the laminar wake flow behind a small hump on a solid wall boundary," International Journal of Non-linear Mechanics, vol. 100, pp. 48-57, 2018.

[2] A. M. Megahed, M. G. Reddy, and W. Abbas, "Modeling of MHD fluid flow over an unsteady stretching sheet with thermal radiation, variable fluid properties and heat flux," Mathematics and Computers in Simulation, vol. 185, pp. 583-593, 2021.

[3] M. G. Reddy and S. A. Shehzad, "Molybdenum disulfide and magnesium oxide nanoparticle performance on micropolar cattaneo-christov heat flux model," Applied Mathematics and Mechanics, vol. 42, no. 4, pp. 541-552, 2021.

[4] A. M. Megahed and M. G. Reddy, "Numerical treatment for MHD viscoelastic fluid flow with variable fluid properties and viscous dissipation," Indian Journal of Physics, vol. 95, no. 4, pp. 673-679, 2021.
[5] M. G. Reddy, P. VIjayakumari, M. V. V. N. L. Sudharani, and K. G. Kumar, "Quadratic convective heat transport of casson nanoliquid over a contract cylinder: an unsteady case," BioNanoScience, vol. 10, no. 1, pp. 344-350, 2020.

[6] M. G. Reddy and K. G. Kumar, "Cattaneo-Christov heat flux feature on carbon nanotubes filled with micropolar liquid over a melting surface: a stream line study," International Communications in Heat and Mass Transfer, vol. 122, Article ID 105142, 2021.

[7] M. G. Reddy, N. K. R, B. C. Prasannakumara, N. G. Rudraswamy, and K. Ganesh Kumar, "Magnetohydrodynamic flow and heat transfer of a hybrid nanofluid over a rotating disk by considering Arrhenius energy," Communications in Theoretical Physics, vol. 73, no. 4, Article ID 045002, 2021.

[8] A. K. Verma, A. K. Gautam, K. Bhattacharyya, and I. Pop, "Entropy generation analysis of Falkner-Skan flow of Maxwell nanofluid in porous medium with temperature-dependent viscosity," Pramana, vol. 95, no. 2, Article ID 69, 2021.

[9] A. K. Pandey, S. Rajput, K. Bhattacharyya, and P. Sibanda, "Impact of metal oxide nanoparticles on unsteady stagnation point flow of the hybrid base fluid along a flat surface," Pramana, vol. 95, no. 1, Article ID 5, 2021.

[10] A. K. Verma, A. K. Gautam, K. Bhattacharyya, and R. P. Sharma, "Existence of boundary layer nanofluid flow through a divergent channel in porous medium with mass suction/injection," Sādhanā, vol. 46, no. 2, Article ID 98, 2021.

[11] A. K. Verma, A. K. Gautam, K. Bhattacharyya, A. Banerjee, and A. J. Chamkha, "Boundary layer flow of non-Newtonian Eyring-Powell nanofluid over a moving flat plate in Darcy porous medium with a parallel free-stream: multiple solutions and stability analysis," Pramana, vol. 95, no. 4, Article ID 173, 2021.

[12] A. Salama, S. Sun, and M. F. Wheeler, "Solving global problem by considering multitude of local problems: application to fluid flow in anisotropic porous media using the multipoint flux approximation," Journal of Computational and Applied Mathematics, vol. 267, pp. 117-130, 2014.

[13] A. Rasheed and M. S. Anwar, "Simulations of variable concentration aspects in a fractional nonlinear viscoelastic fluid flow," Communications in Nonlinear Science and Numerical Simulation, vol. 65, pp. 216-230, 2018.

[14] I. Podlubny, Fractional Differential Equations, Academic Press, New York, NY, USA, 1999.

[15] J. Sabatier, O. P. Agrawal, and J. A. Tenreiro Machado, Advances in Fractional Calculus: Theoretical Developments and Applications in Physics and Engineering, Sprin. Publish. Company, New York, NY, USA, 2007.

[16] Y. Liu, "Solvability of anti-periodic BVPs for impulsive fractional differential systems involving Caputo and Riemann-Liouville fractional derivatives," International Journal of Nonlinear Sciences and Numerical Stimulation, vol. 19, 2018.

[17] D. P. Ahokposi, A. Atangana, and D. P. Vermeulen, "Modelling groundwater fractal flow with fractional differentiation via Mittag-Leffler law," The European Physical Journal Plus, vol. 132, no. 4, Article ID 165, 2017.

[18] A. Atangana and D. Baleanu, "New fractional derivatives with nonlocal and non-singular kernel: theory and application to heat transfer model," Thermal Science, vol. 20, no. 2, pp. 763-769, 2016.

[19] K. Sayevand and K. Pichaghchi, "Analysis of nonlinear fractional $\mathrm{KdV}$ equation based on He's fractional derivative," Nonlinear Sci. Lett. A, vol. 7, pp. 77-85, 2016. 
[20] M. Khan, S. Hyder Ali, and H. Qi, "On accelerated flows of a viscoelastic fluid with the fractional Burgers' model," Nonlinear Analysis: Real World Applications, vol. 10, no. 4, pp. 2286-2296, 2009.

[21] M. Shoaib Anwar and A. Rasheed, "Heat transfer at microscopic level in a MHD fractional inertial flow confined between non-isothermal boundaries," The European Physical Journal Plus, vol. 132, no. 7, Article ID 305, 2017.

[22] L. Wang and J. Wang, "Multiplicity results for degenerate fractional p-Laplacian problems with critical growth," International Journal of Nonlinear Sciences and Numerical Stimulation, vol. 19, no. 2, pp. 215-222, 2018.

[23] J. Zhao, L. Zheng, X. Zhang, and F. Liu, "Unsteady natural convection boundary layer heat transfer of fractional Maxwell viscoelastic fluid over a vertical plate," International Journal of Heat and Mass Transfer, vol. 97, pp. 760-766, 2016.

[24] X.-J. Yang, "General fractional calculus operators containing the generalized Mittag-Leffler functions applied to anomalous relaxation," Thermal Science, vol. 21, pp. S317-S326, 2017.

[25] N. Makris, G. F. Dargush, and M. C. Constantinou, "Dynamic analysis of generalized viscoelastic fluids," Journal of Engineering Mechanics, vol. 119, no. 8, pp. 1663-1679, 1993.

[26] M. S. Anwar and A. Rasheed, "Simulations of a fractional rate type nanofluid flow with non-integer Caputo time derivatives," Computers \& Mathematics with Applications, vol. 74, no. 10, pp. 2485-2502, 2017.

[27] M. Mozafarifard, D. Toghraie, and H. Sobhani, "Numerical study of fast transient non-diffusive heat conduction in a porous medium composed of solid-glass spheres and air using fractional Cattaneo subdiffusion model," International Communications in Heat and Mass Transfer, vol. 122, Article ID 105192, 2021.

[28] M. S. Anwar, "Numerical study of transport phenomena in a nanofluid using fractional relaxation times in Buongiorno model," Physica Scripta, vol. 95, no. 3, Article ID 035211, 2020.

[29] S. E. Ahmed, "Caputo fractional convective flow in an inclined wavy vented cavity filled with a porous medium using Al2O3$\mathrm{Cu}$ hybrid nanofluids," International Communications in Heat and Mass Transfer, vol. 116, Article ID 104690, 2020.

[30] M. S. Anwar, R. T. M. Ahmad, T. Shahzad, M. Irfan, and M. Z. Ashraf, "Electrified fractional nanofluid flow with suspended carbon nanotubes," Computers \& Mathematics with Applications, vol. 80, no. 5, pp. 1375-1386, 2020.

[31] V. J. Ervin, H. Lee, and J. Ruiz-Ramírez, "Nonlinear Darcy fluid flow with deposition," Journal of Computational and Applied Mathematics, vol. 309, pp. 79-94, 2017.

[32] Z. Zhou and D. Liang, "The mass-preserving and modifiedupwind splitting DDM scheme for time-dependent convection-diffusion equations," Journal of Computational and Applied Mathematics, vol. 317, pp. 247-273, 2017.

[33] N. S. Akbar, D. Tripathi, and Z. H. Khan, "Numerical investigation of Cattanneo-Christov heat flux in CNT suspended nanofluid flow over a stretching porous surface with suction and injection," Discrete and Continuous Dynamical Systems-Series S, vol. 11, pp. 595-606, 2018.

[34] A. Aziz, W. Jamshed, and W. Jamshed, "Unsteady MHD slip flow of non Newtonian power-law nanofluid over a moving surface with temperature dependent thermal conductivity," Discrete \& Continuous Dynamical Systems-S, vol. 11, no. 4, pp. 617-630, 2018.

[35] B. J. Kirby, Micro and Nanoscale Fluid Mechanics: Transport in Microfluidic Devices, Cambridge Univ. Press, Cambridge, UK, 2010.
[36] M. Sheikholeslami and D. D. Ganji, "Nanofluid flow and heat transfer between parallel plates considering Brownian motion using DTM," Computer Methods in Applied Mechanics and Engineering, vol. 283, pp. 651-663, 2015.

[37] Y. Liu and Ji-H. He, "Bubble electrospinning for mass production of nanofibers," International Journal of Nonlinear Sciences and Numerical Stimulation, vol. 8, pp. 393-396, 2011.

[38] P. Valipour, H. Zaersabet, M. Hatami, A. Zolfagharian, and S. E. Ghasemi, "Numerical study on polymer nanofibers with electrically charged jet of viscoelastic fluid in electrospinning process," Journal of Central South University, vol. 24, no. 10, pp. 2275-2280, 2017.

[39] G. Wang and J. Zhang, "Thermal and power performance analysis for heat transfer applications of nanofluids in flows around cylinder," Applied Thermal Engineering, vol. 112, pp. 61-72, 2017.

[40] M. Waqas, M. I. Khan, T. Hayat, and A. Alsaedi, "Numerical simulation for magneto Carreau nanofluid model with thermal radiation: a revised model," Computer Methods in Applied Mechanics and Engineering, vol. 324, pp. 640-653, 2017.

[41] S. Nazari, R. Ellahi, M. M. Sarafraz, M. R. Safaei, A. Asgari, and O. A. Akbari, "Numerical study on mixed convection of a non-Newtonian nanofluid with porous media in a two liddriven square cavity," Journal of Thermal Analysis and Calorimetry, vol. 140, no. 3, pp. 1121-1145, 2020.

[42] M. H. Heydari, H. L. Dastjerdi, and M. N. Ahmadabadi, "An efficient method for the numerical solution of a class of nonlinear fractional Fredholm integro-differential equations," International Journal of Nonlinear Sciences and Numerical Stimulation, vol. 19, 2018.

[43] Y. Lin and C. Xu, "Finite difference/spectral approximations for the time-fractional diffusion equation," Journal of Computational Physics, vol. 225, no. 2, pp. 1533-1552, 2007.

[44] R. A. Adams, Sobolev Spaces, Academic Press, New York, NY, USA, 1975.

[45] A. M. Abd El-Lateif and A. M. Abdel-Hameid, "Comment on "Solutions with special functions for time fractional free convection flow of Brinkman-type fluid" by F. Ali et al," The European Physical Journal Plus, vol. 132, no. 9, Article ID 407, 2017.

[46] J. Buongiorno, "Convective transport in nanofluids," Journal of Heat Transfer, vol. 128, no. 3, pp. 240-250, 2006. 